\title{
Modeling of Synchronous Data Streams Processing in the RPC Muon Trigger System of the CMS Experiment
}

\author{
Krzysztof T. Poźniak
}

\begin{abstract}
This paper presents signal synchronization aspects in a large, distributed, multichannel RPC Muon Trigger system in the CMS experiment. The paper is an introduction to normalized structure analysis methods of such systems. The method introduces a general model of the system, presented in a form of a network of distributed, synchronous, pipeline processes. The model is based on a definition of a synchronous data stream and its formal, fundamental properties. Theoretical considerations are supported by a practical application of synchronous streams and processes management. The following processes were modeled and implemented in hardware: window synchronization, derandomization, data concentration and generation of test pulses. There are presented chosen results of the model application in the CMS experiment.
\end{abstract}

Keywords-Synchronization, particle measurements, data acquisition, triggering, measurement system.

\section{INTRODUCTION}

$\mathbf{T}$ HE Resistive Plate Chambers Muon Trigger (RPC MT) [1] is an integral part of the CMS experiment [2] at LHC accelerator in CERN. RPC chambers [3] are positioned in the external part of the CMS detector, where $|\eta|<2.1$ (see Fig. 1). The task of the RPC MT is to provide information to the Global Muon Trigger (GMT). Required data concern four muons, the most energetic ones, originating from the barrel and endcaps regions of the CMS. Search for a muon trajectory is performed every $25 \mathrm{~ns}$ independently for each proton bunch crossing. An algorithm of fast comparison of all registered muon trajectories with preset pattern trajectories has been implemented [1].

Final determination of muon candidates is performed by a synchronous pipeline electronic system of the RPC MT in a several levels of signal processing. These systems may be modeled functionally as a distributed synchronous network of simultaneous pipeline processes. By a process it is understood here an indivisible function of the pipeline processing. A network of processes builds a particular functional layer of the system, or realizes a defined task or a number of combined tasks for the CMS experiment.

Fig. 2 presents a schematic diagram of pipeline processes network used in the RPC MT. Due to large system dimensions and its coherent structure, it is useful to show in Fig. 2 only the most important signal processing functions, not loosing

This work was partially supported by Polish Committee for Scientific Research under grants 115/E-343/SPB/CERN/P-03/DZ 444/2002 and 621/E78/SPB/CERN/P-03/DZ 445/2002.

The Author is with the Institute of Electronic System, Warsaw University of Technology, 00-665 Warsaw, Poland (e-mail: pozniak@ise.pw.edu.pl). the signal transformation idea. Individual processes are symbolized by oval rectangles. The number given at the bottom of oval rectangle is an approximate number of implementation of these particular processes in the whole RPC MT system. Signal connections are symbolized by arrows. The description of arrows shows the total number of transmitted bits for a particular trigger level.

Signals (A) from the RPC chamber strips are initially synchronized (1). The resulting digital signals (B) are compressed (2) from two or three RPC chambers [4]. The compressed data streams (C) are transmitted via optical gigabit links from the CMS detector to the counting room, where they are redistributed (3) to appropriate detection processes of muon trajectories (4). The processors perform, by a comparative method, measurements of the path curvature of muons in a strong magnetic field $(4 \mathrm{~T})$ and further determine the most energetic muons in separated areas of the CMS detector [2]. The final choice of the four most energetic muons $(\mathrm{H})$ for the CMS trigger, is performed in a cascade muon sorter (5-7). An important design assumption for this part of the RPC MT system is a constant latency Ltm for the calculation of the trigger, which equals to 89 LHC clock periods, i.e. $2.225 \mu \mathrm{s}$ [1].

In parallel to the calculation of the trigger decision $(\mathrm{H})$, data acquisition (8) is done for the first level trigger (FLT) of the CMS experiment. After the data concentration (9), full

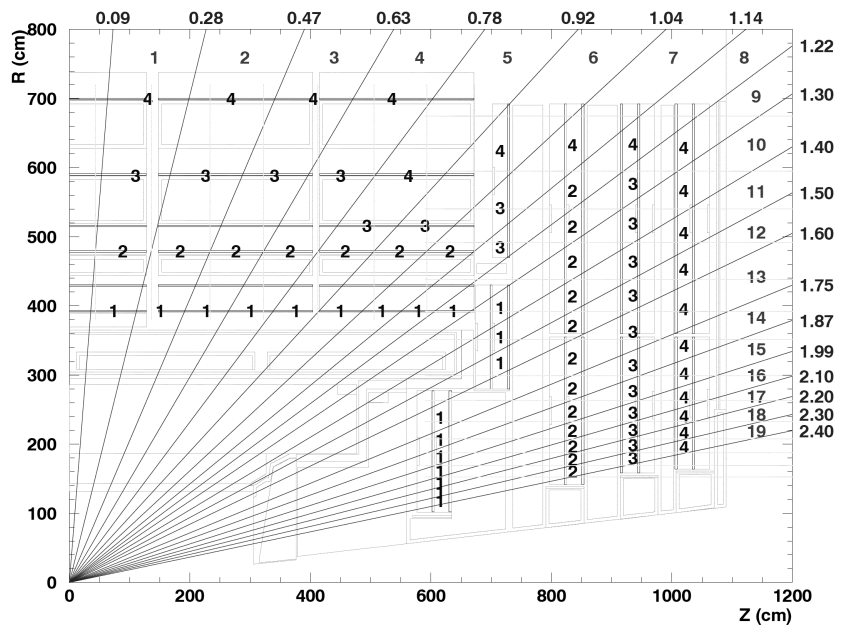

Fig. 1. Arranging of the RPC chambers into towers in the cross section of the CMS (one quarter of the CMS is presented), where $\eta=\arctan (\theta / 2)$. 


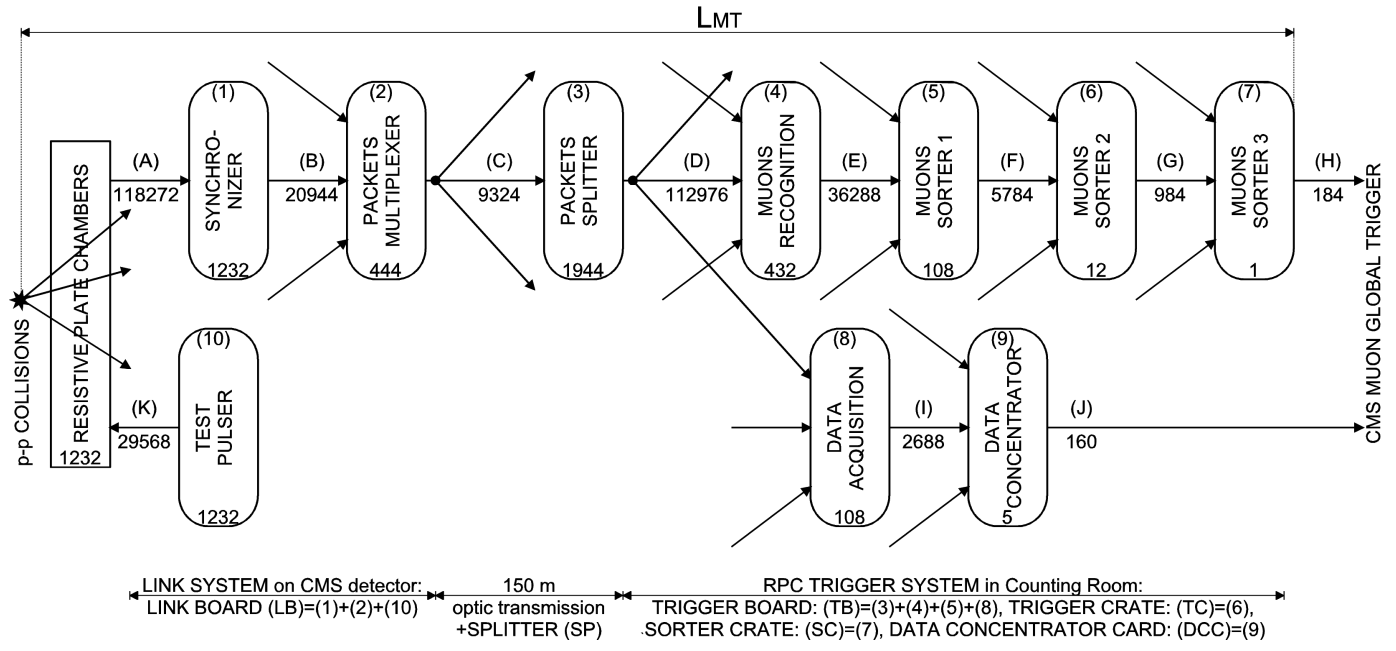

Fig. 2. Schematic stages for processes and signal distribution in the RPC MT.

images of chosen events are temporarily registered $(\mathrm{J})$. They are further selected for research purposes on the next levels of the CMS trigger. Finally accepted events are permanently registered for the off-line analysis. The rest of the events is lost.

Programmable pulsers (10) are connected directly to the analog channels of the RPC detectors $(\mathrm{K})$. They provide testing capabilities for the RPC MT with determined excitation patterns. All channels are tested against errors in the signal processing, synchronization and integration with other CMS sub-systems. Pulsers enable constant monitoring and tuning of individual channel calibration parameters as well as effective and early detection of faults and failures.

In summary, the following characteristics of the RPC MT may be listed (see Fig. 2):

- A pipeline solution stems directly from the system structure; the processes are included into the paths of information distribution and are nodes in the network;

- The whole network works in a synchronous mode;

- The distributed information may be analog or digital;

- The network nodes may play different roles of: flow processes (1),(3), concentration processes (2),(4)-(9) and data source processes (10);

- Network connections link a transmitting node with a receiving node $(\mathrm{B}),(\mathrm{E}-\mathrm{J})$ or with many receiving nodes (multicasting) (C), (D);

- Network node is a logical functional unity and may not be represented as a separate block in the physical hardware realization of the system. For example, the node (7) is, in physical realization, a cascade of nondependent sorting circuits distributed on several PCBs and positioned one next to the other in a single VME crate;

- The node may bridge a number of sub-networks or separate networks.

The above systemization of the characteristics was a foundation to prepare a coherent hardware and software ${ }^{1}$ solutions

\footnotetext{
${ }^{1}$ The synchronization software for the RPC MT is not presented here as it was described in details elsewhere [5], [6].
}

to automatic synchronization of the measurement data distribution in the whole system of the RPC MT. Chapters II and III successively present the models of a stream and a synchronous process. The models were used in the design of the RPC MT. Chapter IV includes short presentations of hardware solution examples. The solutions were implemented in FPGA circuits for particular types of the synchronous processes used in the RPC MT. The experimental results from the RPC MT, obtained during synchronization tests and the first period of the CMS operation were included in chapters V and VI.

\section{SynCHRONOUS DATA STREAM}

A synchronous stream, which distributes information between nodes, is denoted as $\langle\mathbf{C}, \mathbf{I}\rangle$. It can be represented by a set of ordered pairs $\left(c_{m}, i_{m}\right)$ for $m=1,2, . ., M$, while ordering of the pairs fulfills a relation for $i_{m}<i_{m+1}$ :

$$
\begin{array}{r}
\langle\mathbf{C}, \mathbf{I}\rangle \stackrel{\text { def }}{=}\left\{\left(c_{m}, i_{m}\right): c_{m} \in \mathbf{C}, i_{m} \in \mathbf{I} \subseteq \Re,\right. \\
m=1, \ldots, M, M \in \aleph\}
\end{array}
$$

Content elements $c_{m}$ represent real information, distributed by a synchronous stream. In practice, they may be single values or complex, multi-component data structure. Content identifier elements $i_{m}$ determine a domain of the synchronous stream. The synchronous stream of power $M$ is represented graphically in Fig. 3.

There are distinguished two basic kinds of contents identifiers in the RPC MT system:

1) Time identifier, which determines time slot relative to the content element. In a general case, a sequence of time

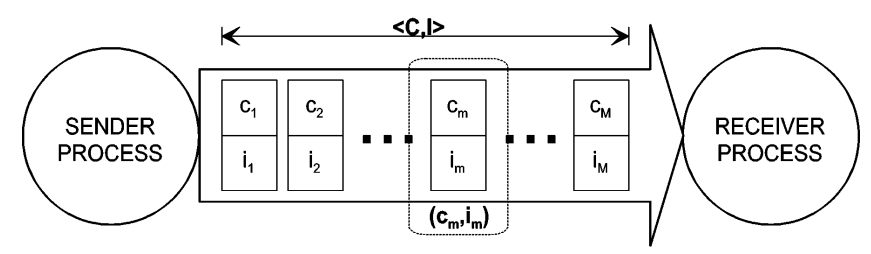

Fig. 3. Symbolic graphical presentation of a synchronous stream. 
identifiers $\mathbf{I}_{\mathbf{T}}=\left\{t_{T 1}, t_{T 2}, \cdots, t_{T M}\right\}$ makes a synchronous time stream $\left\langle\mathbf{C}, \mathbf{I}_{\mathbf{T}}\right\rangle$. This kind of a stream is mainly used in the channels of pipeline signal processing and calculation of the trigger response. The CMS experiment use the time identification associated with bunch crossings. A time interval $T_{A}=1 / f_{A}$ is determined, where $f_{A}$ is the collision frequency:

$$
\forall(1 \leq m \leq M) i_{B m}=m \cdot T_{A}
$$

Synchronous processing of streams $\left\langle\mathbf{C}, \mathbf{I}_{\mathbf{B}}\right\rangle$ is reduced, in practice, to the realization of a finite state automata (e.g. Moore machine or Mealy machine).

2) Event identifier, is a sequence of elements $i_{E m} \in \mathbf{I}_{\mathbf{E}}$, which unambiguous bounds content elements $c_{m} \in \mathbf{C}$ with a particular event in the CMS experiment. This kind of synchronous stream identification is used predominantly in data acquisition channels. The CMS triggers are used for sorting and choice of measured data. After data triggering, the following stream is obtained $\left\langle\mathbf{C}, \mathbf{I}_{\mathbf{T}}\right\rangle$, where the values of event identifiers $\mathbf{I}_{\mathbf{T}}$ are time slots for the successive triggers. The data acquisition process is frequently identified by a single trigger indicator - an event number. This kind of identifier will be indicated as $\mathbf{I}_{\mathbf{E}}$, and the related stream appropriately as: $\left\langle\mathbf{C}, \mathbf{I}_{\mathbf{E}}\right\rangle$.

\section{STREAM SYNCHRONIZATION}

Synchronization between stream elements, which are parts of correlated processes, is a necessary condition for proper work of the pipeline system. Synchronization of elements belonging to two streams $\left\langle\mathbf{C}_{\mathbf{1}}, \mathbf{I}_{\mathbf{1}}\right\rangle$ and $\left\langle\mathbf{C}_{\mathbf{2}}, \mathbf{I}_{\mathbf{2}}\right\rangle$ is, from the definition, determined by a synchronization function $S$, $S: \mathbf{I}_{\mathbf{1}} \rightarrow \mathbf{I}_{\mathbf{2}}$ :

$$
\left\langle\mathbf{C}_{\mathbf{1}}, \mathbf{I}_{\mathbf{1}}\right\rangle \leftrightarrow\left\langle\mathbf{C}_{\mathbf{2}}, \mathbf{I}_{\mathbf{2}}\right\rangle \Leftrightarrow \mathbf{I}_{\mathbf{2}}=S\left(\mathbf{I}_{\mathbf{1}}\right)
$$

where the sign of bidirectional arrow " $\leftrightarrow$ ” is synchronicity relation operator. A reverse synchronization function $S^{-1}$ is:

$$
\begin{array}{r}
\left\langle\mathbf{C}_{\mathbf{1}}, \mathbf{I}_{\mathbf{1}}\right\rangle \leftrightarrow\left\langle\mathbf{C}_{\mathbf{2}}, S\left(\mathbf{I}_{\mathbf{1}}\right)\right\rangle \\
\Leftrightarrow\left\langle\mathbf{C}_{\mathbf{1}}, S^{-1}\left(\mathbf{I}_{\mathbf{2}}\right)\right\rangle \leftrightarrow\left\langle\mathbf{C}_{\mathbf{2}}, \mathbf{I}_{\mathbf{2}}\right\rangle
\end{array}
$$

Several various forms of the synchronization can be distinguished from the definition (3). Fig. 4 presents an example of synchronization for a single element and Fig. 5 for two synchronous streams.

In the case of partial synchronization of streams (synchronization of a single element or group of elements or a sub-stream - part of the main stream) the power of both synchronous streams may be different. The pipeline systems use frequently a sort of synchronization, where the number of not synchronized elements in the streams is small, thus practically negligible. Such a form of streams synchronization is called a quasi-synchronization.

\section{A. Basic Types of Synchronization}

Absolute synchronization for elements of two streams $\left\langle\mathbf{C}_{\mathbf{1}}, \mathbf{I}_{\mathbf{1}}\right\rangle$ and $\left\langle\mathbf{C}_{\mathbf{2}}, \mathbf{I}_{\mathbf{2}}\right\rangle$ determines a special case of definition

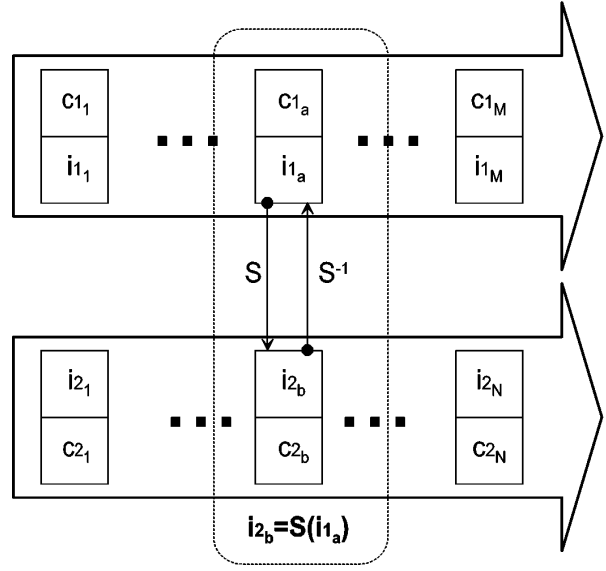

Fig. 4. Synchronization of element $\left(c_{1_{a}}, i_{1_{a}}\right)$ with element $\left(c_{2_{b}}, i_{2_{b}}\right)$ by function $S$.

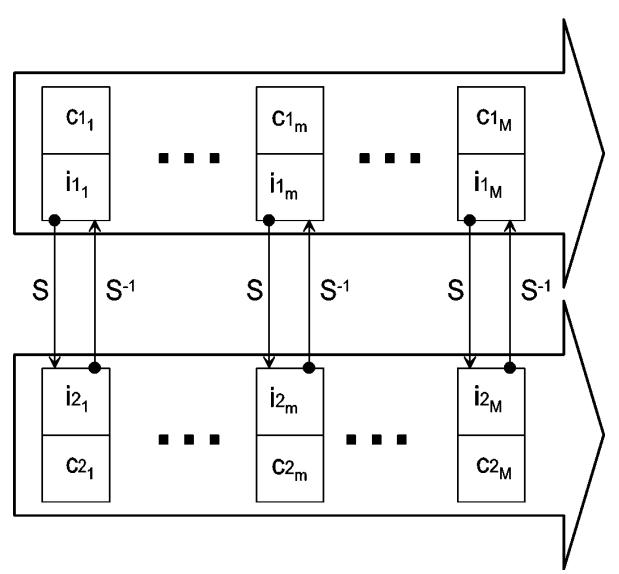

Fig. 5. Synchronization of stream $\left\langle\mathbf{C}_{\mathbf{1}}, \mathbf{I}_{\mathbf{1}}\right\rangle$ with stream $\left\langle\mathbf{C}_{\mathbf{2}}, \mathbf{I}_{\mathbf{2}}\right\rangle$ by function $S$.

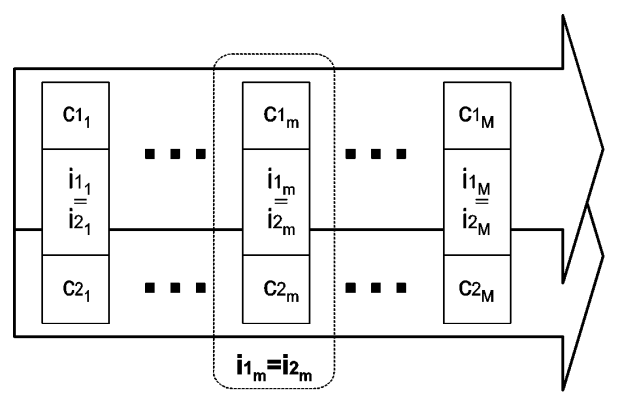

Fig. 6. Absolute synchronization of streams $\left\langle\mathbf{C}_{\mathbf{1}}, \mathbf{I}_{\mathbf{1}}\right\rangle$ and $\left\langle\mathbf{C}_{\mathbf{2}}, \mathbf{I}_{\mathbf{2}}\right\rangle$.

(3) for function $S$ representing equivalence of identifiers (sign as " $\bar{\leftrightarrow}$ "), what also enables mutual exchange of identifiers:

$$
\begin{aligned}
\mathbf{I}_{\mathbf{1}}=\mathbf{I}_{\mathbf{2}} & \Leftrightarrow\left\langle\mathbf{C}_{\mathbf{1}}, \mathbf{I}_{\mathbf{1}}\right\rangle \overline{\bar{\leftrightarrow}}\left\langle\mathbf{C}_{\mathbf{2}}, \mathbf{I}_{\mathbf{2}}\right\rangle \\
& \Leftrightarrow\left\langle\mathbf{C}_{\mathbf{1}}, \mathbf{I}_{\mathbf{2}}\right\rangle \overline{\bar{\leftrightarrow}}\left\langle\mathbf{C}_{\mathbf{2}}, \mathbf{I}_{\mathbf{1}}\right\rangle
\end{aligned}
$$

Streams in the state of an absolute or quasi-absolute synchronization play very important role in information processing. Mutual, logical and numerical operations are possible on the content elements. Graphical representation of the absolute synchronization of two streams $\left\langle\mathbf{C}_{\mathbf{1}}, \mathbf{I}_{\mathbf{1}}\right\rangle$ and $\left\langle\mathbf{C}_{\mathbf{2}}, \mathbf{I}_{\mathbf{2}}\right\rangle$ is shown in Fig. 6. 

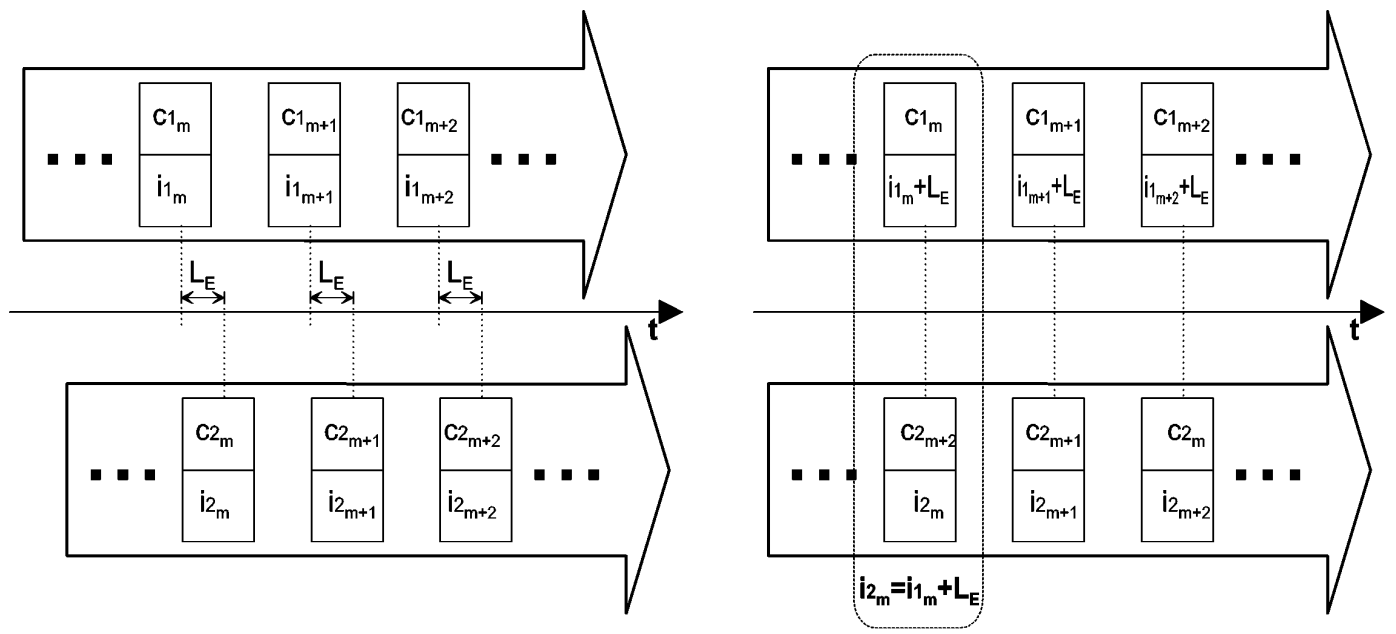

Fig. 7. Equalizing synchronization of a stream $\left\langle\mathbf{C}_{\mathbf{1}}, \mathbf{I}_{\mathbf{1}}\right\rangle$ against a stream $\left\langle\mathbf{C}_{\mathbf{2}}, \mathbf{I}_{\mathbf{2}}\right\rangle$.

\section{B. Synchronization Functions}

In reference to the types of identifiers described in chapter II, there are shown below two synchronization functions $S: \mathbf{I}_{\mathbf{1}} \rightarrow \mathbf{I}_{\mathbf{2}}$ in the pipeline systems for the RPC MT:

1) Equalization Synchronization, which is determined by an equalization of a real parameter $L_{E} \in \mathbf{R}$ added to the value of each identifier of set $\mathbf{I}_{1}$, which in short will be further designated as " $\mathbf{I}_{\mathbf{1}}+L_{E}$ ":

$$
\begin{array}{r}
\left.\quad \mathbf{C}_{\mathbf{1}}, \mathbf{I}_{\mathbf{1}}+L_{E}\right\rangle \leftrightarrow\left\langle\mathbf{C}_{\mathbf{2}}, \mathbf{I}_{\mathbf{2}}\right\rangle \\
\Leftrightarrow \forall(1 \leq m \leq M) i_{1_{m}}+L_{E}=i_{2_{m}}
\end{array}
$$

The inverse function $S^{-1}$ is equivalent to translation of the equalizing synchronization to a second stream. It is determined by the expression:

$$
\begin{array}{r}
\left.\quad \mathbf{C}_{\mathbf{1}}, \mathbf{I}_{\mathbf{1}}\right\rangle \leftrightarrow\left\langle\mathbf{C}_{\mathbf{2}}, \mathbf{I}_{\mathbf{2}}-L_{E}\right\rangle \\
\Leftrightarrow \forall(1 \leq m \leq M) i_{1_{m}}=i_{2_{m}}-L_{E}
\end{array}
$$

and consequently fulfills a relation (4):

$$
\begin{aligned}
& \left\langle\mathbf{C}_{\mathbf{1}}, \mathbf{I}_{\mathbf{1}}+L_{W}\right\rangle \leftrightarrow\left\langle\mathbf{C}_{\mathbf{2}}, \mathbf{I}_{\mathbf{2}}\right\rangle \\
\Leftrightarrow & \left\langle\mathbf{C}_{\mathbf{1}}, \mathbf{I}_{\mathbf{1}}\right\rangle \leftrightarrow\left\langle\mathbf{C}_{\mathbf{2}}, \mathbf{I}_{\mathbf{2}}-L_{W}\right\rangle
\end{aligned}
$$

Fig. 7 presents an example of equalization synchronization of stream elements $\left\langle\mathbf{C}_{1}, \mathbf{I}_{1}\right\rangle$ relative to a stream $\left\langle\mathbf{C}_{2}, \mathbf{I}_{2}\right\rangle$. The values of identifiers $\mathbf{I}_{1}$ and $\mathbf{I}_{2}$ mark positions on the time axis. This kind of synchronization is widely used for the streams determined in time domain $\left\langle\mathbf{C}, \mathbf{I}_{\mathbf{T}}\right\rangle$, where the value of $L_{E}$ parameter is stream latency in the delay line. Fig. 8. presents schematically a practical application of standard delay line

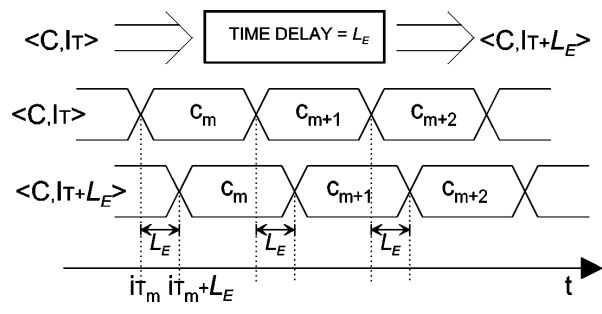

Fig. 8. Application of a delay line for equalizing synchronization. to synchronize two streams. The stream $\left\langle\mathbf{C}, \mathbf{I}_{\mathbf{T}}+L_{E}\right\rangle$ was delayed by $L_{E}$. The content identifiers are modified despite the fact that the content elements of the stream there are delayed.

2) Position Synchronization, which requires determination of $N_{P}$ parameter (belonging to the domain of natural numbers), and which is added to each identifier of set $\mathbf{I}_{\mathbf{1}}$, which will be further denoted as: "I $\mathbf{I}_{\mathbf{1}}[+] N_{P}$ ", with an addition symbol positioned in square brackets (meaning the change of index):

$$
\begin{array}{r}
\left\langle\mathbf{C}_{\mathbf{1}}, \mathbf{I}_{\mathbf{1}}[+] N_{P}\right\rangle \leftrightarrow\left\langle\mathbf{C}_{\mathbf{2}}, \mathbf{I}_{\mathbf{2}}\right\rangle \\
\Leftrightarrow \forall\left(1 \leq m \leq M-N_{P}\right) i_{2_{m}}=i_{1_{m+N_{P}}}
\end{array}
$$

Transfer of synchronization to the second stream is represented by the following transformation:

$$
\begin{array}{r}
\left\langle\mathbf{C}_{\mathbf{1}}, \mathbf{I}_{\mathbf{1}}\right\rangle \leftrightarrow\left\langle\mathbf{C}_{\mathbf{2}}, \mathbf{I}_{\mathbf{2}}[-] N_{P}\right\rangle \\
\Leftrightarrow \forall\left(N_{P} \leq m \leq M\right) i_{1_{m}}=i_{2_{m-N_{P}}}
\end{array}
$$

The transformation represents an inverse function $S^{-1}$ which fulfills the relation (4):

$$
\begin{aligned}
& \left\langle\mathbf{C}_{\mathbf{1}}, \mathbf{I}_{\mathbf{1}}[+] N_{P}\right\rangle \leftrightarrow\left\langle\mathbf{C}_{\mathbf{2}}, \mathbf{I}_{\mathbf{2}}\right\rangle \\
\Leftrightarrow & \left\langle\mathbf{C}_{\mathbf{1}}, \mathbf{I}_{\mathbf{1}}\right\rangle \leftrightarrow\left\langle\mathbf{C}_{\mathbf{2}}, \mathbf{I}_{\mathbf{2}}[-] N_{P}\right\rangle
\end{aligned}
$$

The $N_{P}$ first elements of the first stream and the $N_{P}$ last elements from the second stream are excluded from the synchronization process. This kind of synchronization is frequently used for packet streams $\left\langle\mathbf{C}, \mathbf{I}_{\mathbf{B}}\right\rangle$ (2) or event streams $\left\langle\mathbf{C}, \mathbf{I}_{\mathbf{E}}\right\rangle$. The value of $N$ parameter is equivalent to a delay of stream of $N_{P}$ elements, what was presented in Fig. 9.

Fig. 10 presents schematically a basic application of a $D$ flipflop circuit as a digital delay line, working with the $T_{A}$ clock. A single circuit shifts back the content identifiers of a single position. An equalizing delay may be used for a packet stream $\left\langle\mathbf{C}, \mathbf{I}_{\mathbf{B}}\right\rangle$, on condition that the introduced delay period fulfills the relation (2).

\section{Distribution and Synchronization of Streams in the RPC MT}

A parallel measurement of transverse muons in the Pattern Comparator Processors (PAC) requires a simultaneous 

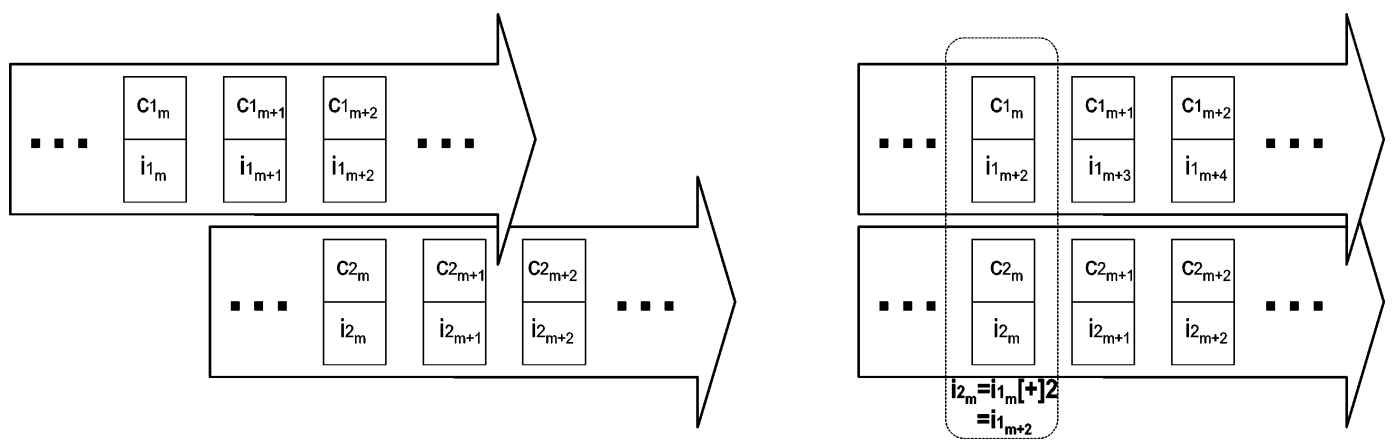

Fig. 9. Position synchronization of a stream $\left\langle\mathbf{C}_{\mathbf{1}}, \mathbf{I}_{\mathbf{1}}\right\rangle$ against a stream $\left\langle\mathbf{C}_{\mathbf{2}}, \mathbf{I}_{\mathbf{2}}\right\rangle$ for $N_{P}=2$.

forwarding of binary detection signals to their inputs from the strips of the RPC detectors (see process (4) in Fig. 2). Finding of four the most energetic muons by means of a cascade sorting from over 5 thousand candidates is realized properly only when the input data are synchronized [5] (processes (5-7) in fig 2). The results of muon measurements are forwarded to the GMT of CMS and are referenced to a particular physical event. Thus, they have to possess a constant latency Lmt (compare Fig. 2). These requirements implicate a full synchronicity in the whole RPC trigger channel. A constant latency is provided during the processes and distribution of synchronous signals.

A serial distribution method for data types $\left\langle\mathbf{C}, \mathbf{I}_{\mathbf{B}}\right\rangle$ (2) was implemented in the RPC MT system for (B-G) stages, in agreement with symbols used in Fig. 2. A $f_{A}=f_{L H C}=40 \mathrm{MHz}$ clock frequency multiplication factor $F$ is defined for each stream. The coefficient determines the serial data stream structure $\left\langle\mathbf{C}_{\mathbf{F}}, \mathbf{I}_{\mathbf{F}}\right\rangle$, calculated in the transmitting process SERIAL. The receiving process DESERIAL reconstructs the input data providing simultaneously a constant delay $L_{T}=L \cdot T_{L H C}$. The parameter $L \in \mathbf{N}$ expresses the latency in the number of LHC clock periods [BX]. The transformation stages in the transmission channel are described by the expression (12):

$$
\begin{array}{r}
\forall(1 \leq m \leq M)\left(c_{m}, i_{B m}\right) \\
\stackrel{\text { SERIAL }}{\longrightarrow}\left\{\left(c_{F m, f}, i_{F m, f}\right), f=\{1, \ldots, F\}\right\} \\
\stackrel{\text { DESERIAL }}{\longrightarrow}\left(c_{m}, i_{B m}+T L\right)
\end{array}
$$

where $F$ successive elements of the serial stream $\left(c_{F m, f}, i_{F m, f}\right)$ are calculated relative to each input element

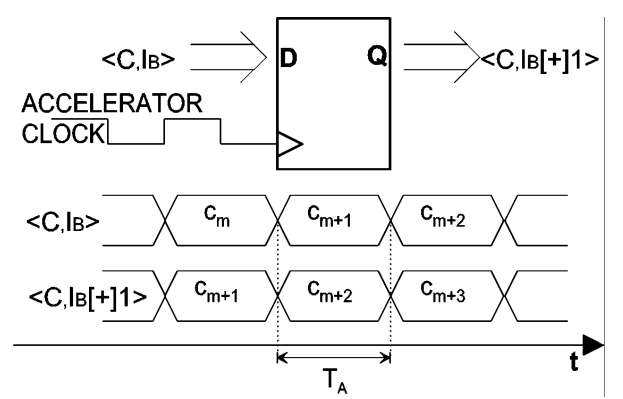

Fig. 10. Usage of a flip-flop circuit of D type to synchronizethe positions of streams. $\left(c_{m}, i_{B m}\right)$ during the SERIAL process, for the values of content identifiers:

$$
\begin{gathered}
\forall\left(i_{B m} \in \mathbf{I}_{\mathbf{B}}\right) \forall(1 \leq f \leq F) \\
i_{F m, f}=i_{B m}+\frac{f-1}{F} T_{L H C}
\end{gathered}
$$

The DESERIAL process has to fulfill the condition:

$$
\begin{array}{r}
\forall\left(i_{B m} \in \mathbf{I}_{\mathbf{B}}\right) \forall(1 \leq f \leq F) \\
\left(i_{B m}+T_{L} \geq i_{F m, f}+t_{P}\right)
\end{array}
$$

where $t_{P}$ is a propagation time for a stream. The following relation of identifier equality $i_{B m}=i_{B S m, 1}$ is fulfilled for $F=1, T_{L}=0$ and $t_{P}=0$. The transmission processes are reduced because the input and the output streams are unconditionally synchronous (5). The condition $L>0$ holds for the remaining cases.

The total distribution of 125 kbits by 4100 synchronous streams is performed with a period of $T_{L H C}$, i.e. every 25 ns. As a consequence, the total net throughput of the whole RPC MT system overcomes $5 \mathrm{~Tb} / \mathrm{s}$. Table I gathers all practically realized implementations. Total gross throughput of the RPC MT is bigger than 5,7 Tb/s and introduces added latency of $32 \mathrm{BX}$. A considerable part of this latency is the propagation time of data from the CMS detector to the electronic modules for stage $(\mathrm{C})$.

The distribution model (12) was used to prepare a universal, parameterized transmission and synchronization module of packet streams [5]. Its general structure was presented in Fig. 11. The module was implemented in FPGA circuits for stages (B-G) in agreement with a diagram presented in Fig. 2. The blocks MUX and DEMUX realize data serialization and deserialization sent with frequency $F C L K=F \cdot f_{L H C}$. The blocks FDELAY and SDELAY provide a programmable setting of the real delays, in the transmission channel respectively, to synchronize the reception of serial data and assure the required latency $L$.

A constant propagation time $t_{P}$ (14) is determined by the latencies introduced successively by the implementation of MUX block, signal distribution in FPGA1, transmission line, signal distribution and DEMUX block implementation in FPGA2:

$$
t_{P}=t_{M}+t_{P 1}+t_{T}+t_{P 2}+t_{D}
$$


TABLE I

IMPLEMENTATION DATA FOR DISTRIBUTION MODULES OF SYNCHRONOUS STREAMS

\begin{tabular}{|c|c|c|c|c|c|c|}
\hline Stage & $\begin{array}{l}\text { Number } \\
\text { of streams }\end{array}$ & $\begin{array}{l}\text { Number of bits in } \\
\text { stream }\end{array}$ & $\begin{array}{l}\text { Transmission } \\
\text { clock [MHz] }\end{array}$ & $\begin{array}{l}\text { Number of trans- } \\
\text { mission lines }\end{array}$ & $\begin{array}{l}\text { Performance } \\
{[\mathrm{Mb} / \mathrm{s}]}\end{array}$ & Latency L [BX] \\
\hline (B) & 1232 & 17 & 40 & 20 & 800 & 1 \\
\hline (C) & 444 & 21 & 1600 & 1 & 1600 & 20 \\
\hline (D) & 1944 & 21 & 320 & 3 & 960 & 3 \\
\hline (E) & 432 & 108 & 320 & 14 & 4480 & 3 \\
\hline (F) & 108 & 68 & 320 & 9 & 2280 & 3 \\
\hline (G) & 12 & 42 & 80 & 24 & 1920 & 2 \\
\hline \multicolumn{2}{|c|}{ Performance /event [1/25 ns]: } & 125596 & \multicolumn{2}{|c|}{ Total performance of the RPC MT system: $[\mathrm{Mb} / \mathrm{s}]$ : } & 5766880 & $\sum=32$ \\
\hline
\end{tabular}

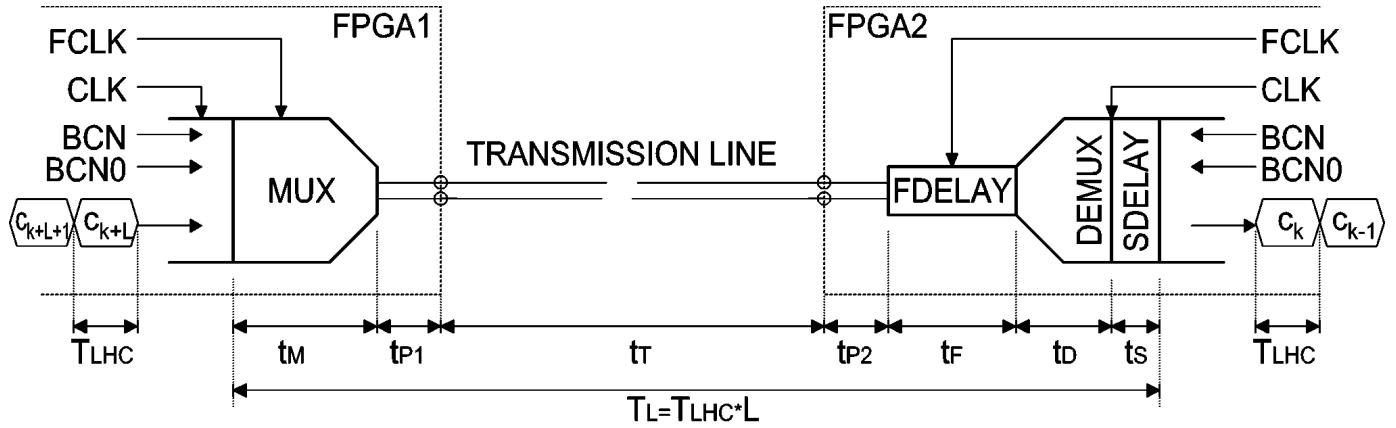

Fig. 11. A general structure of synchronous stream distribution model with a constant latency.

The value of $t_{P}$ delay varies for particular FPGA circuits and with the change of their configuration. Appropriate fitting of the stream delay $T_{L}$ requires fulfilling of the following relation:

$$
T_{L}=t_{P}+T_{L H C}\left(N_{F} S^{-1}+N_{S}\right)+t_{A D J}
$$

where coefficients $N_{F}$ and $N_{S}$, which are changed programmably, determine respectively the delay for blocks FDELAY and SDELAY. Both blocks realize a function of position synchronization (9) for FCLK and CLK clocks. A free parameter $t_{A D J}$ is a supplement of propagation time $t_{P}$ and is contained in a range, open from the right side $\left\langle 0, N_{F} T_{L H C} S^{-1}\right)$. An exemplary realization of the equalization circuit, which eliminates the parameter $t_{P}$ by equalizing synchronization (6) is presented in Fig. 12. D type flip-flop circuits were applied, working at the LHC clock rate. The rising edge of clock signal is forwarding the input data to the flip-flop outputs. This equalizes the time shift $t_{A D J}$ inside a single $T_{L H C}$. Practical solutions have to take into the account clock and data signals jitter and dispersion of the rising time of the signals. These uncertainties complicate technical realization of

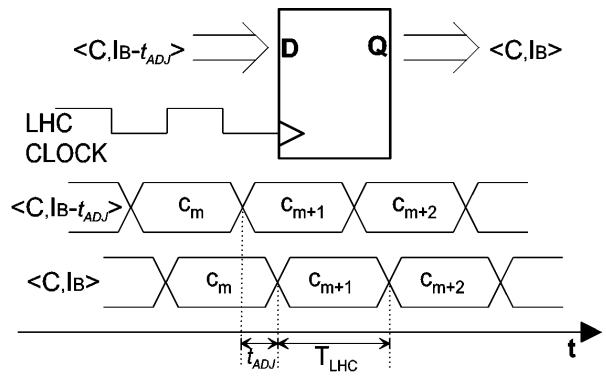

Fig. 12. A general structure of distribution model for synchronous stream of constant latency. the equalization circuit, what is addressed in details in chapter IV-A.

A correct status of the stream synchronization is controlled by a hardware verification of the sent content identifier (BCN and $\mathrm{BCN} 0$ ) in the receiver. The implementation of real content identifiers in the stream transmission is a foundation of a complex monitoring algorithm and automated calibration of the synchronization for the whole RPC MT. This solution considerably increases the system reliability and provides fast localization of irregularities [5].

\section{SyNCHRONOUS PROCESSES}

Synchronous streams form a network of information distribution between processes, or the nodes of distributed pipeline system. Each process realizes a separate part of the pipeline data processing in the system. $K$ input streams $\left\langle\mathbf{C}_{\mathbf{X k}}, \mathbf{I}_{\mathbf{X k}}\right\rangle$, $k=1,2, . ., K$ are transformed to the output stream $\left\langle\mathbf{C}_{\mathbf{Y}}, \mathbf{I}_{\mathbf{Y}}\right\rangle$ :

$$
\left\langle\mathbf{C}_{\mathbf{Y}}, \mathbf{I}_{\mathbf{Y}}\right\rangle=P\left(\left\langle\mathbf{C}_{\mathbf{X} \mathbf{1}}, \mathbf{I}_{\mathbf{X} \mathbf{1}}\right\rangle, \ldots,\left\langle\mathbf{C}_{\mathbf{X K}}, \mathbf{I}_{\mathbf{X K}}\right\rangle\right)
$$

Taking into account the two presented kinds of indexing, one can distinguish the following characteristic forms of streams transformation, shown in Table II.

Transformations of the type $P_{B B}$ are implemented in signal processing channels from detectors (like channel synchronization, compression, etc.), calculation of local triggers (like approximation algorithms for particles trajectories, determination

TABLE II

FORMS OF STREAMS TRANSFORMATIONS

\begin{tabular}{lll}
\hline \hline $\begin{array}{l}\text { Input stream } \\
\left\langle\mathbf{C}_{\mathbf{X k}}, \mathbf{I}_{\mathbf{X k}}\right\rangle \text { with: }\end{array}$ & Output stream $\left\langle\mathbf{C}_{\mathbf{Y}}, \mathbf{I}_{\mathbf{Y}}\right\rangle$ with: \\
packet identifier $\left(\mathbf{I}_{\mathbf{B}}\right)$ & event identifier $\left(\mathbf{I}_{\mathbf{E}}\right)$ \\
\hline packet identifier $\left(\mathbf{I}_{\mathbf{B}}\right)$ & $P_{B B}: \mathbf{I}_{\mathbf{B}} \rightarrow \mathbf{I}_{\mathbf{B}}$ & $P_{B E}: \mathbf{I}_{\mathbf{B}} \rightarrow \mathbf{I}_{\mathbf{E}}$ \\
event identifier $\left(\mathbf{I}_{\mathbf{E}}\right)$ & $P_{E B}: \mathbf{I}_{\mathbf{E}} \rightarrow \mathbf{I}_{\mathbf{B}}$ & $P_{E E}: \mathbf{I}_{\mathbf{E}} \rightarrow \mathbf{I}_{\mathbf{E}}$ \\
\hline \hline
\end{tabular}




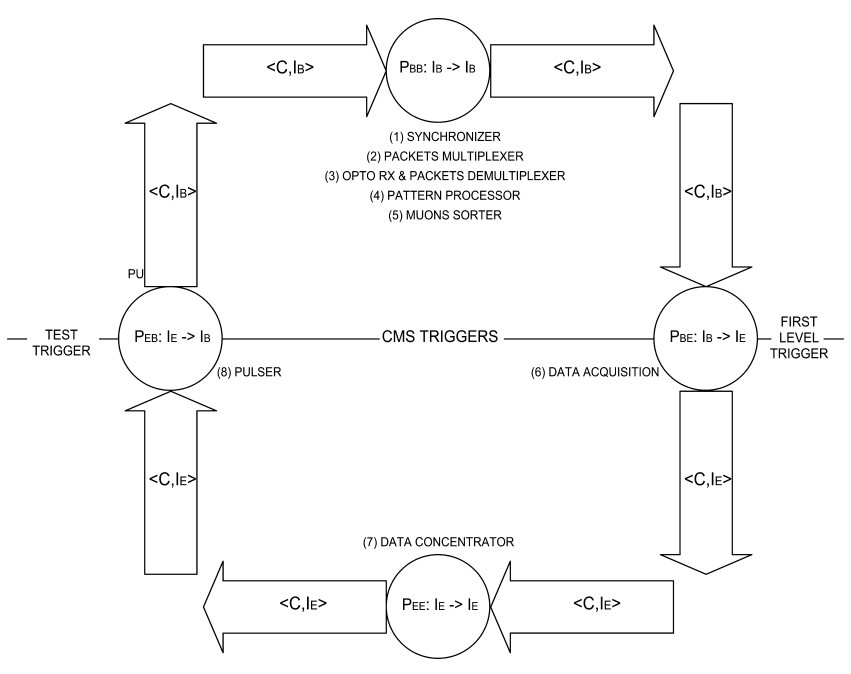

Fig. 13. Transformations of synchronous streams in the RPC MT system.

of deposited energies, etc.) and processing of Global Trigger signals (mainly determination of conditions for the first level trigger).

Transformations of the types $P_{B E}$ and $P_{E E}$ are combined with the data acquisition in the CMS experiment. The initial stage is triggering (derandomization) in processes of $P_{B E}$ type, or extraction of events from the physical data stream. The above processes are synchronous with the trigger (for example, with the Global Trigger). Gathered events, originating from the different detector areas, may be integrated with the usage of processes of type $P_{E E}$. Transformations of the type $P_{E B}$ are generally applied in test, synchronizing and diagnostic processes. They enable generation of test signal sequences, based on the previously prepared events.

Synchronous packets $\left\langle\mathbf{C}, \mathbf{I}_{\mathbf{B}}\right\rangle$ and events $\left\langle\mathbf{C}, \mathbf{I}_{\mathbf{E}}\right\rangle$ streams (chapter II) were implemented in the RPC MT system. There were used all types of the transformations integrated with the CMS experiment triggering, from Table II. Fig. 13 shows, in a schematic way, stream transformations relative to the structure of the system presented in Fig. 2. All processes combined with RPC trigger calculation (1)-(5) are of the $P_{B B}$ type. Only synchronous streams $\left\langle\mathbf{C}, \mathbf{I}_{\mathbf{B}}\right\rangle$, or the ones identified by the number of bunch crossing in LHC accelerator were applied. Streams $\left\langle\mathbf{C}, \mathbf{I}_{\mathbf{E}}\right\rangle$ identified by the trigger number of the first level (FLT) of the CMS experiment were used in the processes of event registration (6) and (7). The derandomization process transforms a stream $\left\langle\mathbf{C}, \mathbf{I}_{\mathbf{B}}\right\rangle$ to $\left\langle\mathbf{C}, \mathbf{I}_{\mathbf{E}}\right\rangle$ synchronously with the FLT. An inverted synchronous transformation with the test trigger is realized during the test process (8).

Further part of the chapter presents appropriate examples of process realization for various transformation types of synchronous streams for the RPC MT of the CMS experiment.

\section{A. Window Synchronization for Signals from the RPC Cham- bers}

Window synchronization of signals from the RPC chambers is an example of equalization synchronization of two input data streams in a process of $P_{B B}$ type. Fig. 14 shows a time diagram of this process. A rising edge of $100 \mathrm{~ns}$ pulse

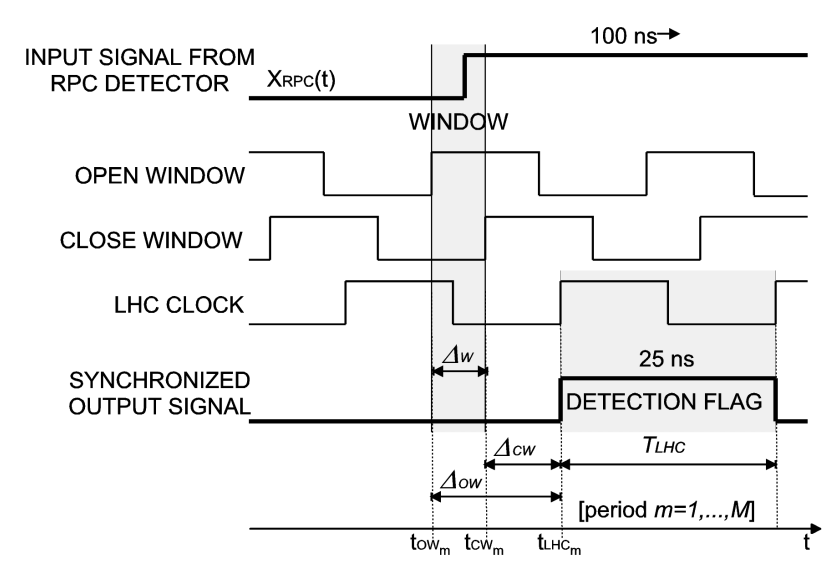

Fig. 14. General time dependencies in the synchronization processfor the RPC MT.

$X_{R P C}(t)$ is synchronized. The edge marks a moment of minimally ionized particle passage through the RPC detector [1]. The rising edge of clock signal OPEN WINDOW marks the beginning of the window time slot. The rising edge of signal CLOSE WINDOW marks the end of the window time slot. These signals are shifted in time relative to the accelerator clock LHC CLOCK by $\Delta_{O W}$ and $\Delta_{C W}$ respectively. Time duration of the window is $\Delta_{W}=\Delta_{O W}-\Delta_{C W}$. The window width is confined by the period of LHC accelerator clock, which is $T_{L H C}=25 \mathrm{~ns}$. The input signal $X_{R P C}(t)$ is registered for a while when the window is opened $\left(t=t_{O W m}\right)$ and closed $\left(t=t_{C W m}\right)$. According to the relation (1) one obtains binary input streams for the process, respectively for successive moments of window opening $\left\langle\mathbf{C}_{\mathbf{O W}}, \mathbf{I}_{\mathbf{O W}}\right\rangle$ and window closing $\left\langle\mathbf{C}_{\mathbf{C W}}, \mathbf{I}_{\mathbf{C W}}\right\rangle$. The streams are indexed with a variable $m=1, \ldots, M$ :

$$
\begin{array}{r}
\left\langle\mathbf{C}_{\mathbf{O W}}, \mathbf{I}_{\mathbf{O W}}\right\rangle=\left\{\left(c_{O W m}=X_{R P C}\left(i_{O W m}\right),\right.\right. \\
\left.\left.i_{O W m}=t_{O W m}=m \cdot T_{L H C}-\Delta_{O W}\right)\right\} \\
\left\langle\mathbf{C}_{\mathbf{C W}}, \mathbf{I}_{\mathbf{C W}}\right\rangle=\left\{\left(c_{C W m}=X R P C\left(i_{C W m}\right),\right.\right. \\
\left.\left.i_{C W m}=t_{C W m}=m \cdot T_{L H C}-\Delta_{C W}\right)\right\}
\end{array}
$$

To do a logical operation of the detection of rising edge of the input signal an initial equalization synchronization (6) is done during the window duration $\left(\Delta_{W}\right)$ for both input streams, by delaying the stream $\left\langle\mathbf{C}_{\mathbf{O W}}, \mathbf{I}_{\mathbf{O W}}\right\rangle$ of factor $\Delta_{W}$ :

$$
\left\langle\mathbf{C}_{\text {ow }}, \hat{\mathbf{I}}_{\text {OW }}\right\rangle \leftrightarrow\left\langle\mathbf{C}_{\text {OW }}, \mathbf{I}_{\text {OW }}-\Delta_{W}\right\rangle
$$

A stream $\left\langle\mathbf{C}_{\mathbf{O W}}, \hat{\mathbf{I}}_{\mathbf{O W}}\right\rangle$ is in the state of absolute synchronization with a stream $\left\langle\mathbf{C}_{\mathbf{C W}}, \mathbf{I}_{\mathbf{C W}}\right\rangle$. According to the relation (5), a common sequence of identifiers $\mathbf{I}_{\mathbf{C W}}$ is obtained for both streams:

$$
\left\langle\mathbf{C}_{\mathbf{O W}}, \hat{\mathbf{I}}_{\mathbf{O W}}\right\rangle \overline{\bar{\leftrightarrow}}\left\langle\mathbf{C}_{\mathbf{C W}}, \mathbf{I}_{\mathbf{C W}}\right\rangle \Leftrightarrow \hat{\mathbf{I}}_{\mathbf{O W}}=\mathbf{I}_{\mathbf{C W}}
$$

Calculation of a binary stream of detection of rising signal edges inside the window $\Delta_{W}$ leads to the stream 


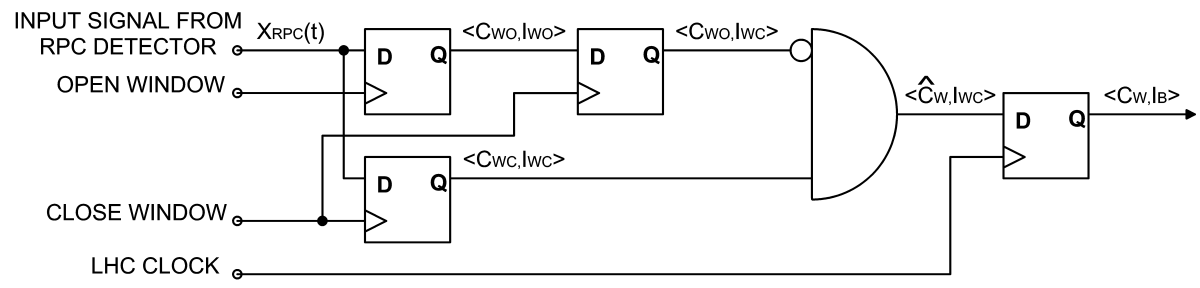

Fig. 15. Digital realization of the window synchronization process.

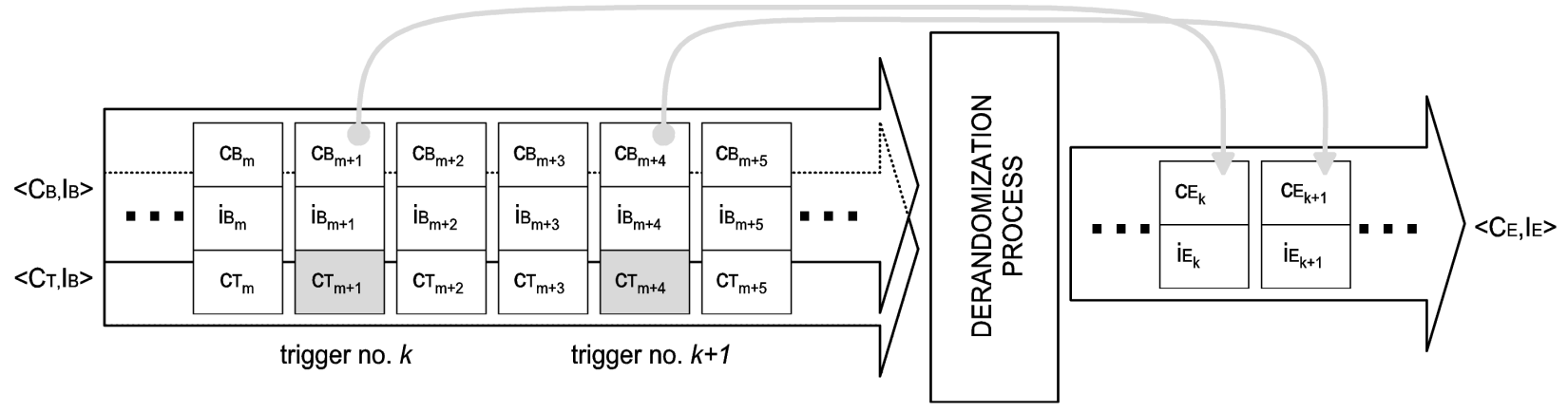

Fig. 16. Derandomization process.

$$
\begin{aligned}
& \left\langle\hat{\mathbf{C}}_{\mathbf{W}}, \mathbf{I}_{\mathbf{C W}}\right\rangle: \\
& \quad\left\langle\hat{\mathbf{C}}_{\mathbf{W}}, \mathbf{I}_{\mathbf{C W}}\right\rangle=\left\{\left(\hat{c}_{W m}=\overline{s_{O W m}} \wedge s_{C W m}, i_{C W m}\right)\right\}
\end{aligned}
$$

where by an upper dash line “-” an operation of logical negation is denoted, and by " $\wedge$ " a logical multiplication.

The result of window synchronization process is the generation, during the closest period of LHC CLOCK, of a single positive logical state signal denoted as DETECTION FLAG. The output binary packet stream $\left\langle\mathbf{C}_{\mathbf{W}}, \mathbf{I}_{\mathbf{B}}\right\rangle$, from the synchronization process, is obtained by delaying of the stream $\left\langle\hat{\mathbf{C}}_{\mathbf{W}}, \mathbf{I}_{\mathbf{C W}}\right\rangle$ by a factor $-\Delta_{C W}$ :

$$
\begin{array}{r}
\left\langle\mathbf{C}_{\mathbf{W}}, \mathbf{I}_{\mathbf{B}}\right\rangle=\left\langle\hat{\mathbf{C}}_{\mathbf{W}}, \mathbf{I}_{\mathbf{C W}}-\Delta_{C W}\right\rangle \\
=\left\{\left(c_{W m}=\hat{c}_{W m}, i_{B m}=t_{L H C}=m \cdot T_{L H C}\right)\right\}
\end{array}
$$

The values of time identifiers, respective to the successive rising edges of clock signal, have consequence in a digital implementation of the window synchronization process, what was presented in Fig. 15.

The above debated process consists, in reality, from two stages of synchronization. During the first stage, a normalized synchronization of the rising edge of a physical signal $X_{R P C}(t)$ is performed relative to the rising edge of LHC CLOCK signal, via a proper choice of two parameters $\Delta_{O W}$ and $\Delta_{C W}$. The possibility of normalization stems from an important rule valid all over the experiment: all particle bunch crossings are strictly synchronous with the CMS clock. During the second stage, a packet output stream is calculated $\left\langle\mathbf{C}_{\mathbf{W}}, \mathbf{I}_{\mathbf{B}}\right\rangle$, where the number of packet is determined by a free parameter $m$. A stream of not-normalized synchronization, relative to the global packet identifier of the experiment (Bunch Crossing Number - BCN), is obtained. Further stages of the signal processing introduce a normalization to obtain the coherent data set for particular event registered in different areas of the whole detector.

\section{B. Synchronous Derandomization with the First Level Trigger of the CMS Experiment}

Derandomization of a chosen event, on the level of the whole CMS experiment, is described by a transformation $P_{D E R A N}$ (23) realized in a process of $P_{B E}$ type. Only data which form a stream of successive events $\left\langle\mathbf{C}_{\mathbf{E}}, \mathbf{I}_{\mathbf{E}}\right\rangle$, synchronously with the binary packet stream of experiment trigger $\left\langle\mathbf{C}_{\mathbf{T}}, \mathbf{I}_{\mathbf{B}}\right\rangle$ are taken from the input stream $\left\langle\mathbf{I}_{\mathbf{B}}, \mathbf{I}_{\mathbf{B}}\right\rangle$ of packet type (2):

$$
\begin{array}{r}
\left\langle\mathbf{C}_{\mathbf{E}}, \mathbf{I}_{\mathbf{E}}\right\rangle=P_{D E R A N}\left(\left\langle\mathbf{I}_{\mathbf{B}}, \mathbf{I}_{\mathbf{B}}\right\rangle,\left\langle\mathbf{C}_{\mathbf{T}}, \mathbf{I}_{\mathbf{B}}\right\rangle\right) \\
\Leftrightarrow \forall\left(c_{T m}=1\right) \exists\left(c_{E k}=c_{B m}, i_{E k}=k\right), k=1,2, \ldots
\end{array}
$$

A synchronization between both input streams is provided by their common identifier $\mathbf{I}_{\mathbf{B}}$. It was assumed that the trigger signal is represented by value 1 , i.e. a condition $c_{T m}=1$ is investigated for successive elements of content sequence $m=1,2, . ., M$ of the stream $\left\langle\mathbf{C}_{\mathbf{T}}, \mathbf{I}_{\mathbf{B}}\right\rangle$. The number of trigger determines the event identifier, thus the quantity of the stream $\left\langle\mathbf{C}_{\mathbf{E}}, \mathbf{I}_{\mathbf{E}}\right\rangle$ responds to the whole number of triggers, i.e. $k=$ $1,2, \ldots$. A graphical representation of the derandomization for two triggers was presented in Fig. 16.

Triggered elements of content (here: $c_{k+1}$ and $c_{k+4}$ ) are transferred successively to the stream of events and identified with the current number of trigger, while the rest of data is lost. During the derandomization process, an unconditional exchange of packet identifiers for event identifiers takes place. Lack of the synchronization between the input streams leads to attributing wrong data to the events. A proper reconstruction of the events in the detector is, thus, impossible. 


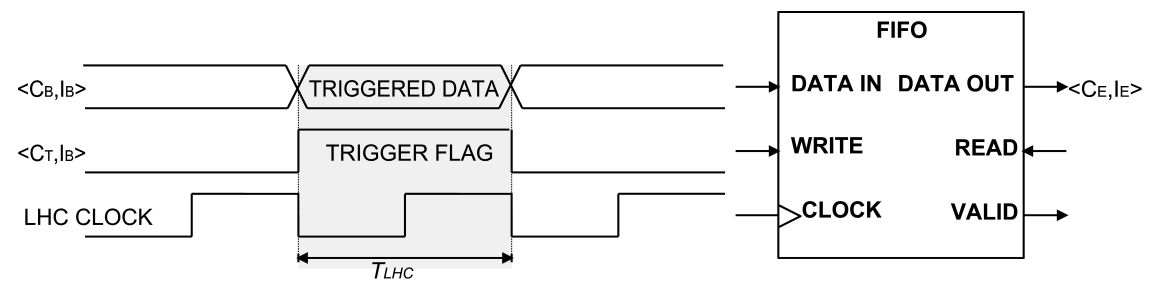

Fig. 17. Digital realization of derandomization process.

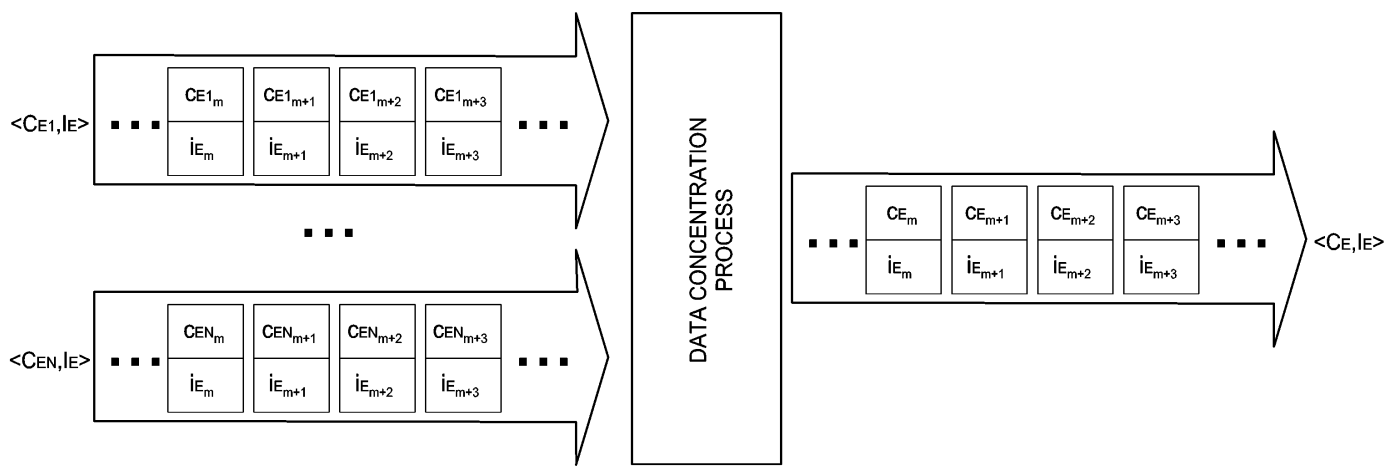

Fig. 18. A process of data stream concentration.

The stream $\left\langle\mathbf{C}_{\mathbf{E}}, \mathbf{I}_{\mathbf{E}}\right\rangle$ is identified with a free parameter $k$. A stream of non-normalized synchronization is obtained relative to the global event identifier for the experiment (Event Number). Data integration from nondependent streams of the events requires the synchronization.

A digital implementation of the derandomization process is presented in Fig. 17. Data are input to the FIFO queue, synchronously with the rising edge of LHC CLOCK signal. The data writing processes is controlled directly by the trigger signal. Data are gathered in the queue and form successive elements of a new stream $\left\langle\mathbf{C}_{\mathbf{E}}, \mathbf{I}_{\mathbf{E}}\right\rangle$. They are successively taken, at the condition of a valid flag VALID and forwarded to the further steps of the data concentration. Length of the queue is respectively correlated with the allowed frequency of experiment trigger and the throughput of further processes, to avoid overfilling. Buffer overfilling leads to data loss, synchronization loss and fatal errors in the data acquisition system.

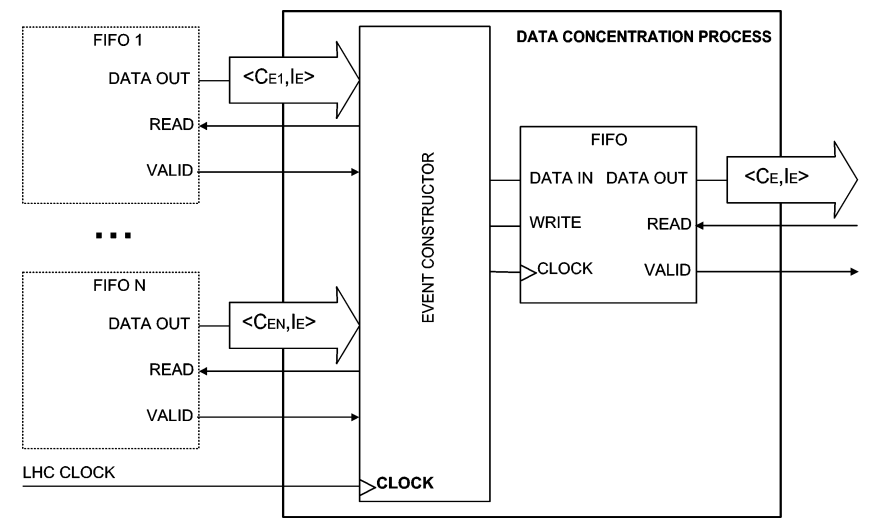

Fig. 19. Digital realization of data concentrator.

\section{Data Concentration at the FLT}

Obtaining the full image of the event, chosen by the FLT of CMS, requires a coherent integration of information from over 100 million nondependent readout channels. Particular event streams are subject to a multistage data concentration. A general relation of a single data concentration stage for $n=1, \ldots, N$ input event streams $\left\langle\mathbf{C}_{\mathbf{E n}}, \mathbf{I}_{\mathbf{E}}\right\rangle$ to an output event stream $\left\langle\mathbf{C}_{\mathbf{E}}, \mathbf{I}_{\mathbf{E}}\right\rangle$ is described by the following transformation $P_{C O N C}$ :

$$
\begin{aligned}
& \left\langle\mathbf{C}_{\mathbf{E}}, \mathbf{I}_{\mathbf{E}}\right\rangle=P_{C O N C}\left(\left\langle\mathbf{C}_{\mathbf{E} \mathbf{1}}, \mathbf{I}_{\mathbf{E}}\right\rangle, \cdots,\left\langle\mathbf{C}_{\mathbf{E N}}, \mathbf{I}_{\mathbf{E}}\right\rangle\right) \\
\Leftrightarrow & \forall\left(c_{E m} \in \mathbf{C}_{\mathbf{E}}\right) c_{E m}=f_{C O N C}\left(c_{E 1 m}, \ldots, c_{E N m}\right)
\end{aligned}
$$

Synchronization of all streams is provided by a common identifier $\mathbf{I}_{\mathbf{E}}$. A common event construction function $f_{C O N C}$ operates on content elements of an identical identifier. The same identifier is ascribed to the result of this function. A graphical representation of this process is presented in Fig. 18. All elements of the input streams $\left\langle\mathbf{C}_{\mathbf{E} \mathbf{1}}, \mathbf{I}_{\mathbf{E}}\right\rangle, \cdots,\left\langle\mathbf{C}_{\mathbf{E N}}, \mathbf{I}_{\mathbf{E}}\right\rangle$ and the output stream $\left\langle\mathbf{C}_{\mathbf{E}}, \mathbf{I}_{\mathbf{E}}\right\rangle$ are identified by a free parameter $m$. Loss of synchronization in the input streams leads to improper data concatenation originating from different physical phenomena. The output stream has a non-normalized synchronization against the global experiment event identifier (Event Number) identified by a free parameter $m$.

A solution for digital implementation of the data concentration process was presented in Fig. 19. This solution assumes that the input streams are forwarded from FIFO queues. The concentrator may directly cooperate with derandomization processes (Fig. 17) or may form own cascade of concentrators. EVENT CONSTRUCTOR realizes function $f_{C O N C}$ separately for each successive event. Initially event data, are taken from all the input streams. Next, an algorithm of event 


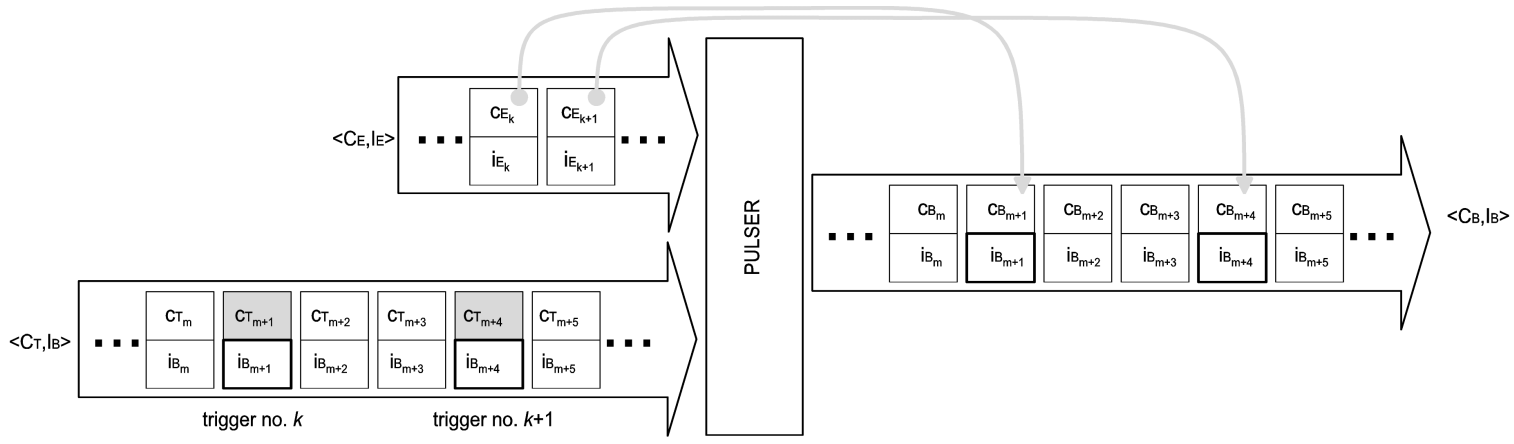

Fig. 20. Process of generation of test pulses.

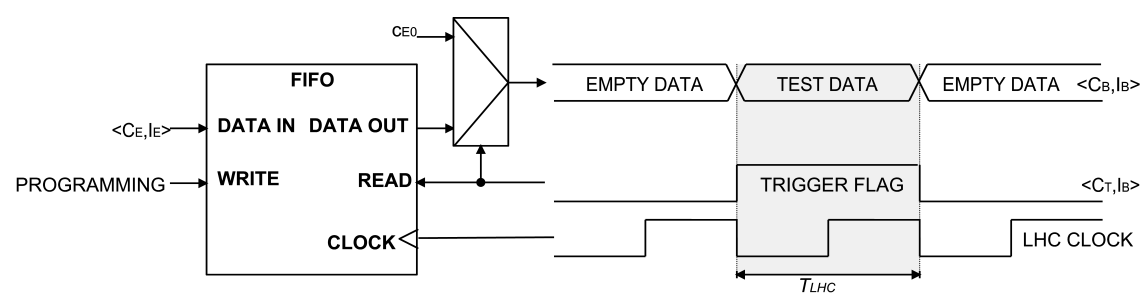

Fig. 21. Digital realization of test pulser.

constructor is performed, which results in the generation of a common output element. Similarly to the derandomization process (chapter IV-B), it is forwarded to the FIFO queues and forms a stream $\left\langle\mathbf{C}_{\mathbf{E}}, \mathbf{I}_{\mathbf{E}}\right\rangle$. Control signals VALID inform EVENT CONSTRUCTOR of the data positioned in the input queues. READ signals enable selective choice of the successive events.

\section{Test Pulse Generation}

Test signal patterns are forwarded to the inputs of ana$\log$ electronics FRONT-END of the RPC chambers in form of synchronously generated pulses. The synchronization is maintained by the test trigger of the CMS experiment. This process is described by a transformation $P_{G E N}$ (25). The pattern signals are contained in the content elements of the event stream $\left\langle\mathbf{C}_{\mathbf{E}}, \mathbf{I}_{\mathbf{E}}\right\rangle$. The patterns are introduced to the output packet test stream $\left\langle\mathbf{I}_{\mathbf{B}}, \mathbf{I}_{\mathbf{B}}\right\rangle$, synchronously with the packet binary trigger stream $\left\langle\mathbf{C}_{\mathbf{T}}, \mathbf{I}_{\mathbf{B}}\right\rangle$. The remaining content elements are designated as empty $\left(c_{B 0}\right)$ :

$$
\begin{array}{r}
\left\langle\mathbf{I}_{\mathbf{B}}, \mathbf{I}_{\mathbf{B}}\right\rangle=P_{G E N}\left(\left\langle\mathbf{C}_{\mathbf{E}}, \mathbf{I}_{\mathbf{E}}\right\rangle,\left\langle\mathbf{C}_{\mathbf{T}}, \mathbf{I}_{\mathbf{B}}\right\rangle\right) \\
\Leftrightarrow\left(\forall\left(c_{T m}=1\right) \exists\left(c_{B m}=c_{E k}, k=1,2, \ldots\right)\right) \\
\vee\left(\forall\left(c_{T m}=0\right) \exists\left(c_{B m}=c_{B 0}\right)\right)
\end{array}
$$

A transformation process of the type $P_{E B}$ is carried out for the input stream of signal patterns $\left\langle\mathbf{C}_{\mathbf{E}}, \mathbf{I}_{\mathbf{E}}\right\rangle$ to the output test stream $\left\langle\mathbf{I}_{\mathbf{B}}, \mathbf{I}_{\mathbf{B}}\right\rangle$. Synchronization of the trigger stream and the output stream provides a common packet identifier $\mathbf{I}_{\mathbf{B}}$. Fig. 20 presents a graphical representation of the process of generation of test pulses. An unconditional exchange of event identifiers $\mathbf{I}_{\mathbf{E}}$ for packet identifiers $\mathbf{I}_{\mathbf{B}}$ takes place during the process of test pulse generation. In the example shown in Fig. 20, single elements of the output streams $c_{B m+1}$ and $c_{B m+4}$ are subject $c_{E k}$ and $c_{E k+1}$ respectively. Lack of synchronization between the elements of input streams leads to improper test data in the output stream. A hardware realization of the test pulse generation for the RPC MT was presented in Fig. 21. A FIFO circuit was used to gather the test data stream (signal PROGRAMMING) and output of the elements synchronously with the trigger signal of the CMS experiment. The multiplexer module is controlled by a trigger signal and switches respective test and empty data.

\section{Synchronization Tests of the RPC MT With CMS MUON TRIGGER}

The presented model of synchronous data streams distribution and processing was implemented in the whole channel of the RPC MT. The implementation, performed in a form of parameterized functional blocks, bases on Cyclone and StratixII chips by Altera and on Spartan3 chip by Xilinx.

Since the second half of 2006, till the end of 2008, particular blocks of the RPC MT were successively subject to the following operations:

1) installation in the CMS experiment: VME crates with LB boards are installed on the detector and crates with TB boards and sorters in the Counting Room;

2) integration with the RPC detectors and remaining subsystems of the CMS;

3) testing of the RPC MT in the full operational environment of the CMS control.

The core of the testing environment are Global Runs of the CMS experiment with participation of most of the subsystems. The RPC MT system participated in 2006 in the Magnet Test and Cosmic Challenge (MTCC) [5]. The field obtained in the CMS magnet was $3.8 \mathrm{~T}$.

This chapter presents the results of the RPC MT performed during a Global Run in November 2007. There were used the RPC chambers from 6 sectors of the barrel, together 36 chambers. An electronic system was used consisting of 81 

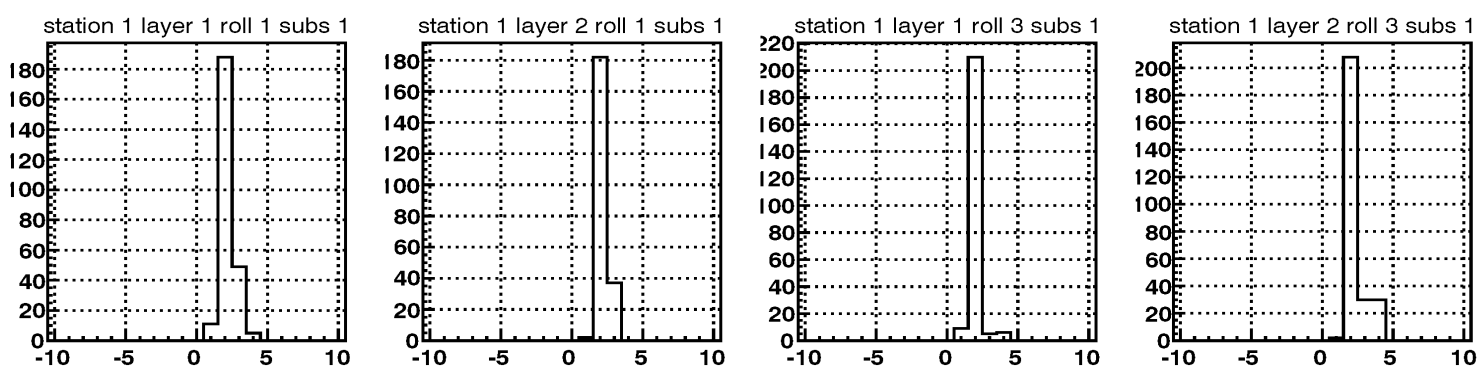

station 2 layer 1 roll 1 subs 1
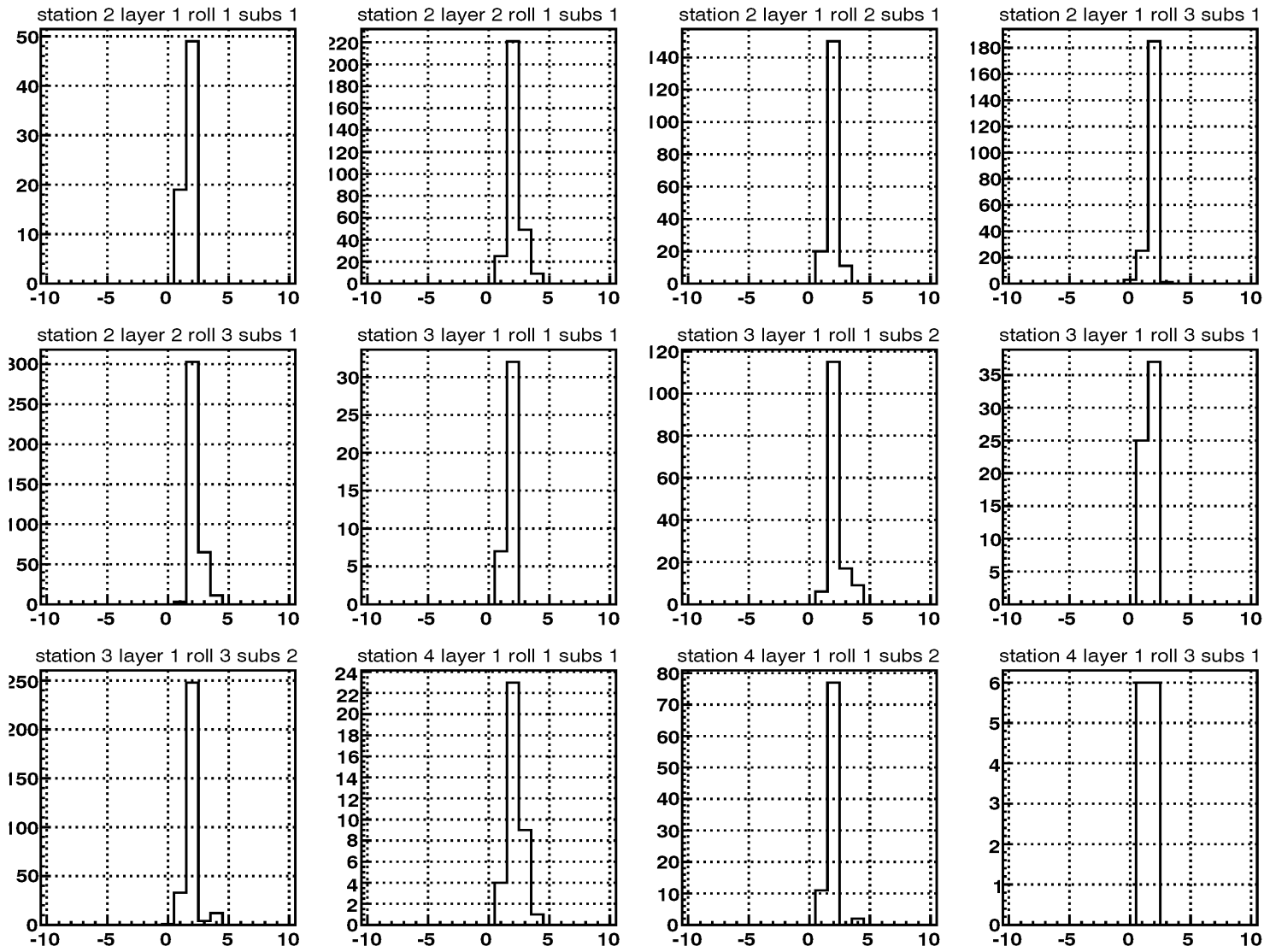

Fig. 22. Time positions of signals from the RPC chambers relative to trigger signal generated by PAC, based on the original signals.

LB81 LB modules, 30 optical links, 4 TB boards situated in two TC crates, and a final TS crate for muon sorting (Fig. 2). The RPC MT was integrated with the GMT and Global Data Acquisition. The RPC provided input data for the triggering process and enabled muon signals registration synchronously with the experiment Global Trigger.

The RPC trigger was generated under the condition that a certified muon trajectory was discovered. The certified trajectory means that it is registered by at least three out of six chamber layers during the same BX. The requirement was to obtain from $3 / 6$ to $6 / 6$ coincidences of signals. The obtained frequency of the trigger was of the order of teen Hz. The triggers were in time coincidence with other triggers generated by the second muon system based on Drift Tube Chambers (DT). A coincidence of such two data facts mean, that the RPC MT generated proper and confirmed physical triggers. The triggers were related strictly to a muon passage through the used part of the detector. If the triggering process is performing properly, it means that the following synchronization conditions (analyzed theoretically above) were fulfilled:

- Choice of individual parameters $\Delta_{O W}$ and $\Delta_{C W}$ for synchronization windows of the signals from the RPC chambers (par. IV.A);

- Obtaining of absolute position synchronization (par. III.B) of packet streams of $\left\langle\mathbf{C}, \mathbf{I}_{\mathbf{B}}\right\rangle$ type, by the choice of delay values for particular streams distributed successively between the processes (2)-(3)-, .., -(7), in agreement with the numbering in Fig. 2;

- Obtaining of nominal latency $L_{M T}$ for the RPC MT system (Fig. 2), which provides appropriate decision coincidence of RPC with the decisions of other muon detectors (DT) which participate in the decision of the GMT.

Fig. 22 presents a family of histograms of time position for signals from RPC for a single sector in correlation with the own trigger of RPC. Bin value on position 2 is related with 


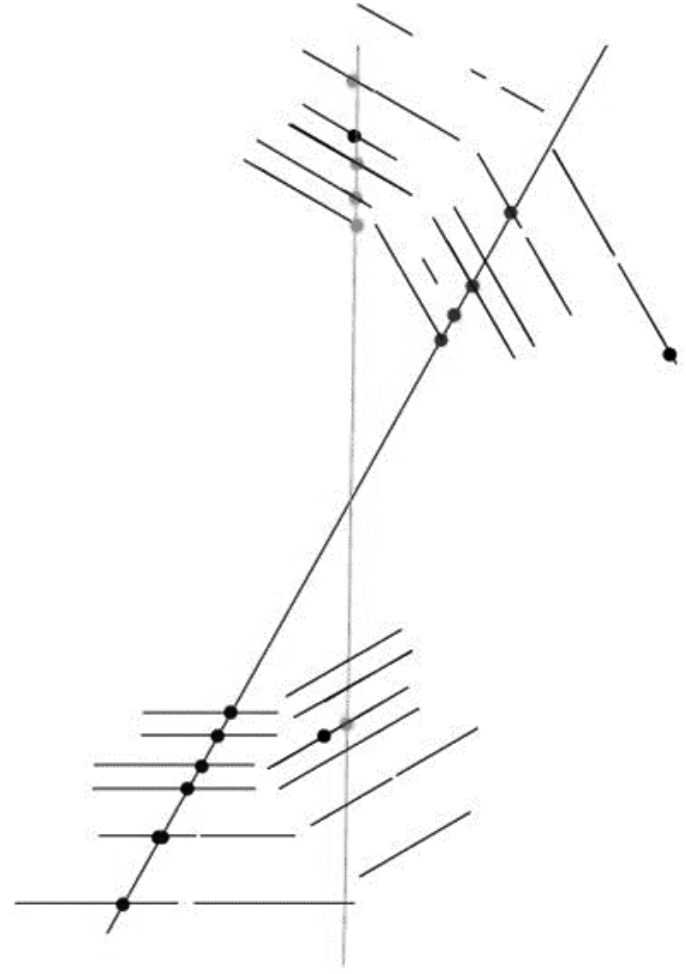

Fig. 23. Cosmic muons in the RPC detector. The drawing presents a crosssection via one of the circles of the barrel in a plane perpendicular to the beam pipe.

the $\mathrm{BX}$, where the trigger was generated. The fact that the majority of signals is concentrated in the $\mathrm{BX}$ related to the trigger is a direct proof of the proper signal synchronization by the LB and that the signal, were transmitted with no errors to the TB, where they were properly read out by the DAQ. Improper setting of the window parameters, improper position synchronization would result in the migration of the response to other $\mathrm{BX}$ positions. The fluctuations observed on the histograms have physical origin in the dispersion of the response time by the chamber, registration of secondary cluster signals or from so called an "after pulse" signal.

The full chain of the data acquisition process requires the following synchronization sub-processes:

- Position synchronization (par. III.B) between processes (3)-(8) according to the numbering in Fig. 2;

- Synchronization of the CMS trigger signal for the derandomization process (8) according to Fig. 2. A proper delay has to be chosen for data from the packet streams (chapter IV-B);

- Concentration of the event streams of $\left\langle\mathbf{C}_{\mathbf{E}}, \mathbf{I}_{\mathbf{E}}\right\rangle$ type in process (9) according to Fig. 2.

Fig. 23 presents an example of registered trajectories of cosmic muons. The strips set to straight traces of muons in the data readout for triggered events, because the CMS magnet was switched off during these measurements.

Registered signals from the RPC chambers (short parallel lines) are marked with points. Muon trajectories (long straight line) are calculated from the data numerically. The signals which do not fit to the calculated trajectories are the own noise of the RPC chambers. A perfect time correlation (the same $\mathrm{BX}$ ) of many signals from various different chambers is again a strong proof of good synchronization of the signals distributed in the RPC. It is also a proof of good synchronization in the whole RPC MT system with the CMS experiment trigger. The above debated case used the DT.

\section{RPC MT SYSTEM SYNCHRONIZATION DURING THE TEST CMS RUNS}

The synchronization procedure of the RPC MT consists of two stages:

1) Setting of hardware parameters which are written to the data base. The values of these parameters are obtained during the hardware tests of the system. These tests have to be performed only in the case of introducing changes to the FPGA circuits configuration (new firmware);

2) Activation of the automatic synchronization procedure for optical fiber links. The procedure was implemented in the FPGA circuits. It sets appropriate parameters for the transmitted data synchronization. Data are sent by GOLs and received by TLK2501 circuits. The global synchronization signal, distributed by TTC system, is used for procedure initialization. Application of the above solution shortened the synchronization process from several minutes (when using PC procedures) to several $\mathrm{ms}$. As a result, the synchronization procedure was included into the automatic configuration of the CMS experiment.

Synchronization status of the RPC MT is monitored online for each bunch crossing (each $25 \mathrm{~ns}$ ) directly inside an FPGA circuit. The synchronization process uses the following data: the compliance verification of the $\mathrm{BX}$ number sent by the transmitter with the $\mathrm{BX}$ number in the receiver; analogous check of BCN0 signals in the receiver and transmitter; control sum check in the received data. An example of the on-line monitoring of work status of the optical fiber links is presented in Fig. 24. Discovery of any lack in the data conformity causes automatic switching off of a particular transmission channel. The channel is switched off for the whole duration of the erroneous data and the error counter is incremented as a result. The above solution prevents generation of false triggers by the RPC MT and registration of false data.

The synchronization verification of the RPC MT was performed during the activation of the LHC. A single proton bunch was directed to the collimators which were $100 \mathrm{~m}$ distant from the CMS detector. A great number of particles were produced flying via the CMS during a period of a few ns. The results in Fig. 25 present the activity distribution of the RPC chamber strips against the nominal BX. The distance of the chambers from the interaction point contained in a single circle is approximately the same. Thus, the particles cross the chambers practically in the same time. The distribution of chamber activity in a single circle is focused in two BXs. This is a direct proof of the correct and accurate synchronization of the signals from the chambers. A shift of the maximum of the distribution against the central circle (no. 0) indicates that the synchronization is correct inside the whole barrel. 

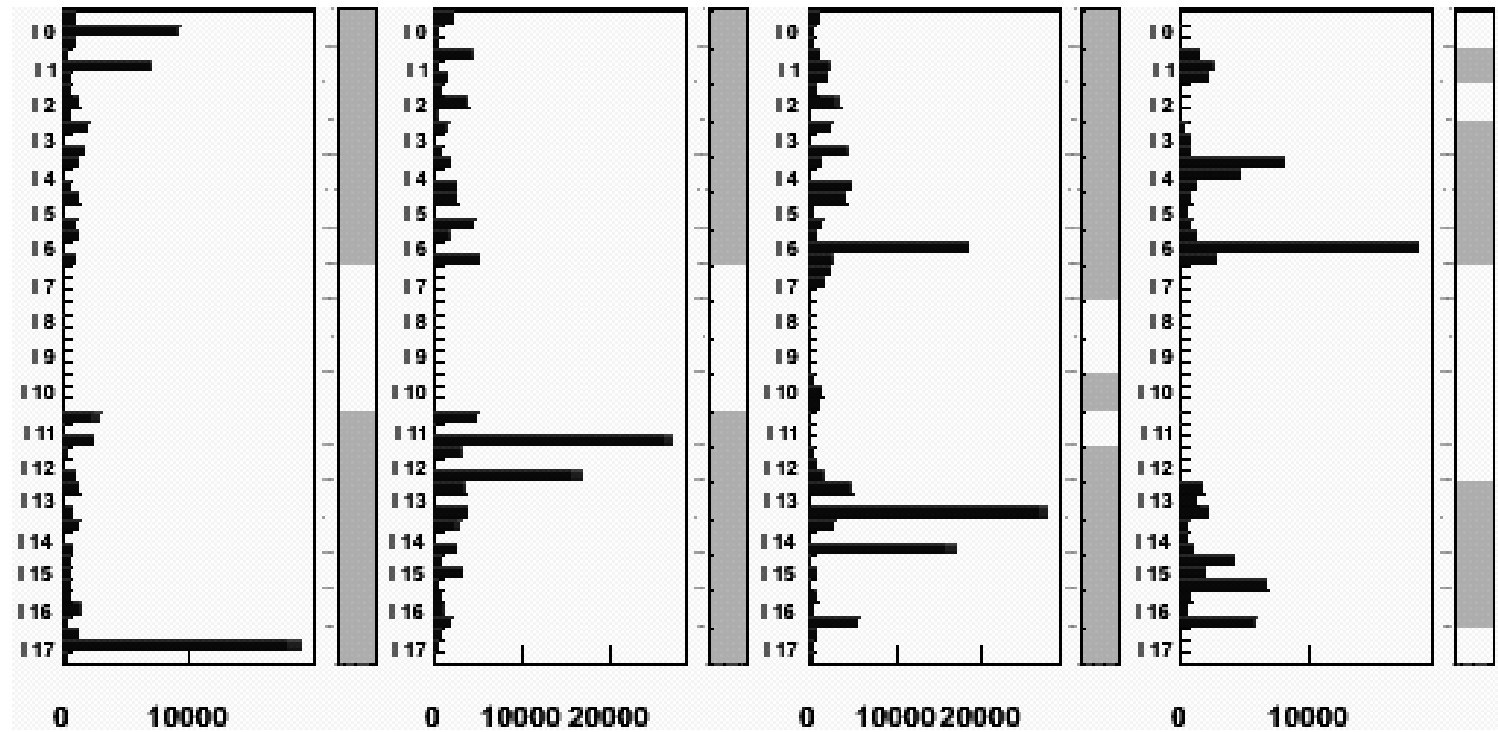

Fig. 24. The chart presents an example monitoring of four optical links. The horizontal bars present the rates [Hz] of non-empty optical data frames transmitted via the links The light grey fields on the right of a histogram present the proper status of the optical links, the white color denotes, that the link is OFF [6].

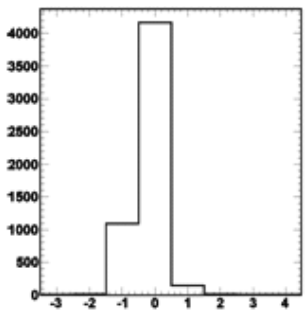

Wheel no. -2

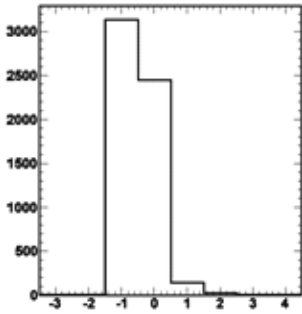

Wheel no. -1

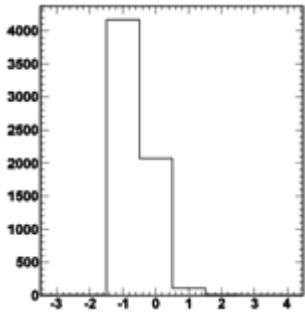

Wheel no. 0

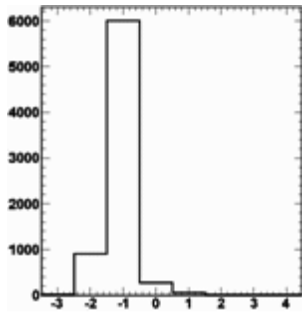

Wheel no. 1

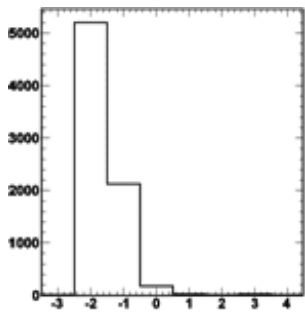

Wheel no. 2

Fig. 25. RPC MT system synchronization status inside the barrel. Splash analysis No. Run121993. BX=0 is a nominal crossing of the splash.

The above configuration of the RPC MT system synchronization was used during the first ever period of the $p-p$ collisions in the LHC. Fig. 26 presents the first case with production of the muon. It was registered in the CMS experiment by the Tracking Detector and Muon Detectors: RPC and DT. The event was properly discovered by the RPC MT. The signals were registered in four RPC chambers in a single bunch crossing. The RPC trigger was produced in the proper $\mathrm{BX}$, in line with the bunch crossing.

\section{CONCLUSIONS}

Proper work of a large, distributed, measurement system, which is the RPC MT requires a precise and stable synchronization. The real RPC MT system has a complex structure and is composed of hundreds and thousands of separate electronic blocks distributed over large areas. Each block realizes particular synchronous processes in the area of measurements, data acquisition, data concentration, fast numerical and logical signal processing, signal and system monitoring and diagnostics. Electrical and optical links are stretched between the modules. They enable fast, synchronous, noiseless and reliable measurement and control data distribution.

The presented analysis approach to the big, distributed measurement system for the RPC MT, as to a network of synchronous processes and data streams, enabled forming a foundation for a homogeneous functional model. This model, for the first time, can be effectively digitized and analyzed numerically. Each of the processes in the model realizes a particular transformation function on the input data streams. Different kinds of data streams, applied in real systems, are represented by a unified form of a synchronous data stream, but of different kinds. The stream identifier defines its domain and enables determination of the synchronization state against other streams.

There were emphasized two efficient forms of stream representations in the RPC MT system. They are packet and data. There were debated a few forms of stream transformations synchronous to the CMS clock. Stream transformation examples, with the corresponding hardware implementations, were given for the RPC MT.

Application of the model to formally describe data streams distribution and synchronization in a networked measurement system has practically no consequence on the size of the hardware layer, thus, further increasing reliability, reducing manufacturing effort, decreasing costs, more efficiently using the ever increasing hardware memory and calculation resources, etc. In a considerable part of practical solutions, the stream identifiers do not have a real representation. They are determined relatively by a parameter which, among others, 


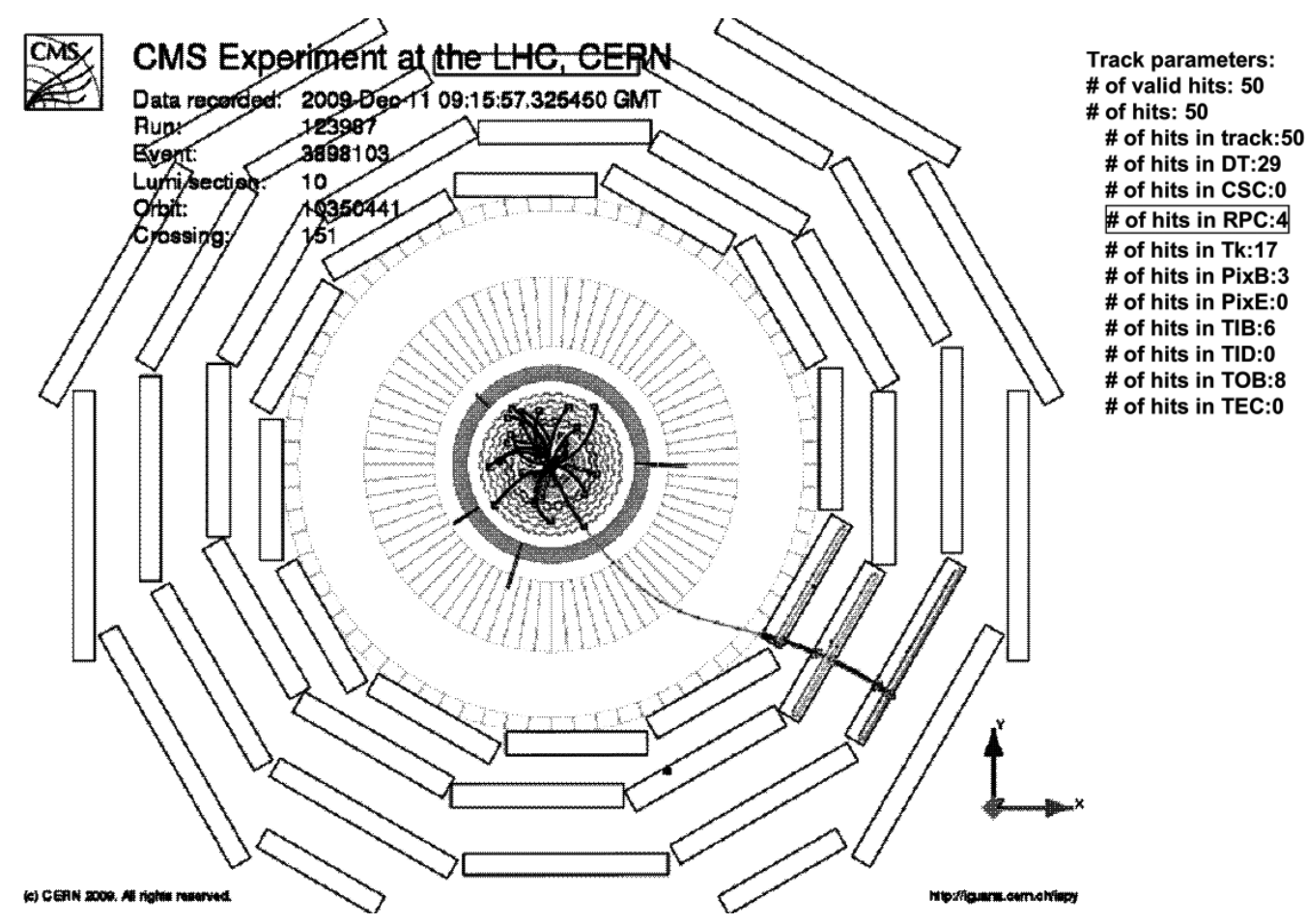

Fig. 26. The first case with production of the muon.

counts rising edges of the experiment clock (chapters IV-A, IV-B and IV-D) or data position in the FIFO memory (chapters IV-B and IV-D). They strictly determine a character of the synchronous stream, and in consequence directly indicate for efficient methods of its synchronization and processing in the system. In this sense, the suggested modeling approach to a large networked measurement system is an efficient tool for designers and users alike. The solutions were positively verified during several MTCC tests in 2006-2007 in CERN (chapter V) and experiment runs in 2009 (chapter VI).

The described synchronization method was successfully applied in the RPC Technical Muon Trigger of the CMS experiment [7].

\section{ACKNOWLEDGMENT}

The author would like to thank warmly all persons and institutions, due to which this work was made possible. This includes:

- the Warsaw CMS Group from the Faculty of Physics, Warsaw University, under the leadership of professor Jan Krolikowski;
- the Warsaw ELHEP Group from the Institute of Electronic Systems, Warsaw University of Technology, under the leadership of professor Ryszard S. Romaniuk.

\section{REFERENCES}

[1] M. Felcini, "The trigger system of the CMS experiment," Nucl. Instr. and Meth. A, vol. 598, no. 1, pp. 312-316, 2009.

[2] S. Chatrchyan et al., "The CMS experiment at the CERN," Journal of Instrumentation, vol. 3, no. S08004, 2008

[3] M. Abbrescia et al., "The RPC system for the CMS experiment at the LHC" Nucl. Instr and Meth. A, vol 508, pp. 137-141, 2003.

[4] M. Górski, M. Kudla, and K. T. Poźniak, "Resistive plate chamber (RPC) based muon trigger system for the CMS experiment data compression/decompression system," Nucl. Instr. and Meth. A, vol. 419, pp. 701-706, 1998.

[5] K. Bunkowski et al., "Synchronization methods for the PAC RPC trigger system in the CMS experiment," Measurement Science and Technology, Institute of Physics, London, vol. 18, pp. 2446-2455, 2007.

[6] K. Bunkowski, "Optimization, synchronization, calibration and diagnostic of the RPC PAC muon trigger system for the CMS detector," Ph.D. dissertation, Institute of Experimental Physics, University of Warsaw, 2009.

[7] R. T. Rajan et al., "A configurable tracking algorithm to detect cosmic muon tracks for the CMS-RPC based technical trigger," Nucl. Instr. and Meth. A, vol. 602, pp. 792-795, 2009. 